\title{
On the numerical modelling of the VLF Trimpi effect
}

\author{
David Nunn \\ Department of Electronics and Computer Science, Southampton University, \\ Southampton SOI7 1BJ, U.K.
}

(Received in final form 11 March 1996; accepted 15 March 1996)

\begin{abstract}
This work is concerned with the numerical modelling of the well known Trimpi effect, in which transient perturbations of amplitude and phase appear in subionospheric VLF transmissions. These perturbations are due to scattering from night-time D region irregularities or LIE's (localised ionisation enhancements), which result from electron density increases due to precipitation from lightning whistlers. A theoretical and numerical technique is developed for modelling the scattering process. The formalism is based on a multi-modal treatment of an assembly of point scatterers, and is subject to the Born weak scattering approximation. The VLF propagation code MODEFNDR is used to determine the total E field incident on each element of the scattering volume. Using the conductivity tensor the effective induced current field in the LIE may be calculated. MODEFNDR is used again to find the total scattered field radiated by the current field induced in the LIE. The technique does not require that the scale length of the LIE is many wavelengths long, and the theory predicts strong intermodal scattering. The model is applied to the paths NWC-Dunedin, Omega(Australia)-Dunedin and NPM, Hawaii-Palmer, Antarctica. The agreement with observations is good. (C) 1997 Elsevier Science Ltd. All rights reserved
\end{abstract}

\section{INTRODUCTION}

The VLF 'Trimpi' effect was first reported in a classical paper by Helliwell et al. (1973). VLF transmission on the paths from transmitters NAA and NSS to Eights and Byrd stations in Antarctica were studied. Transient changes in received amplitude up to $6 \mathrm{~dB}$ were recorded. The time duration of these perturbations was observed to be some tens of seconds. In another classic paper, Lohrey and Kaiser (1979) reported transient VLF phase perturbations of up to 8 degrees in magnitude on the path NWC (Australia) to Dunedin (New Zealand).

The Trimpi effect is concerned with the subionospheric propagation of monochromatic (CW) VLF signals, usually at night. Isolated perturbations of received amplitude and phase occur, typical maximum amplitudes being about $6 \mathrm{~dB}$ for amplitude and 10 degrees for phase. The onset of such perturbations occurs within a few seconds, and the perturbation decays away in a time scale of $10-40 \mathrm{~s}$. The time scales of the phase and amplitude disturbances may in fact be different. In this paper we present one example of a VLF Trimpi event, on NAA transmissions, observed at the British Antarctic Survey base at Faraday (see Fig. 1). This is a 'classic' Trimpi event in which the amplitude perturbation is negative and the phase perturbation is positive. This example also clearly exhibits the sudden onset and then exponential decay, as well as the different time constants for the amplitude and phase disturbances. The 'classic' Trimpi is given the label $(-+)$ and is what one expects from simple theory. Long overwater paths in which there is a dominant TM mode tend to produce $(-+)$ Trimpis, but there is always a statistical scatter. Trimpis that have positive amplitude and phase perturbations $(++)$, positive amplitude and negative phase $(+-)$ and negative amplitude and phase (- ) arc all not uncommon. Short paths and paths dominated by multi-modal effects tend to produce Trimpis scattered in all four quadrants.

Helliwell et al. (1973) quickly identified the cause of the Trimpi effect. The perturbations in received VLF signal are due to scattering from perturbation patches in the $\mathrm{D}$ region of the ionosphere. These patches (or LIE's-localised enhancements of ionisation) are mainly due to precipitation of energetic electrons $(\sim \mathrm{kcV})$ by VLF lightning whistlers. Lightning spherics radiate an impulse in the VLF band which propagates in a ducted mode through the magnetosphere. Dispersion results in the classical 'whistler' waveform. Energetic electrons undergo cyclotron resonant interaction in the equatorial zone as a result of which some particles are thrown into the loss cone and precipitate out into the lower ionosphere in the height range 50$90 \mathrm{~km}$. The result is a localised region or patch of enhanced electron density, located in the $\mathrm{D}$ region 

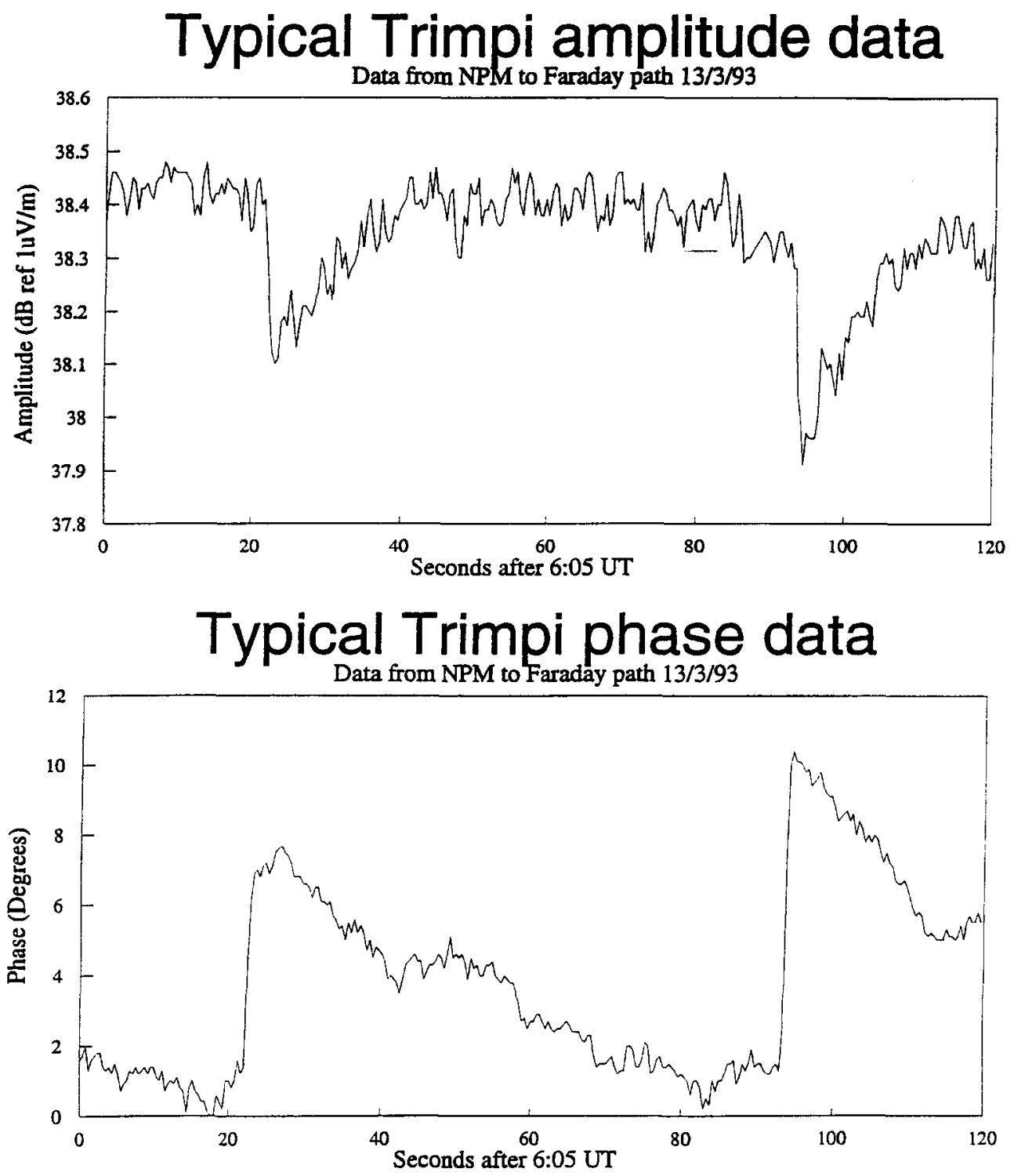

Fig. 1. Record of a typical 'Trimpi' observed at Faraday, Antarctica. The VLF transmitter is NAA. The plots show amplitude and phase disturbance. This is a classical $(-+)$ Trimpi. Note the different decay times for amplitude and phase disturbance.

close to the field line appertaining to the duct in question. A single lightning strike may excite multiple ducts giving multiple patches, and the precipitation flux may have considerable variation or structure across the cross section of the duct. Not all lightning strikes will produce patches if the VLF radiation does not couple into nearby ducts or if the particle instability level or particle distribution function are unfavourable. As well as producing patches within a few $100 \mathrm{~km}$ of itself, a lightning discharge may also produce patches near the magnetically conjugate point. This can occur either due to reflection of the 
VLF whistler or, for example near the S Atlantic anomaly, due to mirroring of particles at the Northern end of the field line and their subsequent precipitation at the Southern end.

The transient $\mathrm{D}$ region enhancements of electron density are not of themselves directly measurable, and there is no agreement on their typical size or structure. Analysis of observations so far points towards a scale size of order $100 \mathrm{~km}$, since patches much smaller than this will not give an observable Trimpi. There is some evidence of elliptically shaped patches orientated with the major axis in the magnetic $\mathrm{E}-\mathrm{W}$ direction. One has to assume that there will be a variety of shapes and sizes, with the distinct possibility of multiple patches. The enhancement of electron density set up by a burst of precipitating electrons will of course decay away due to collisions, the recombination rate being faster at low altitudes. As the patch decays away its vertical structure will change, tending to move to higher altitudes.

The first task of this paper will be to analyse the scattering process, which is a fascinating theoretical topic in its own right. We will then progress to a numerical modelling of the process which is as accurate as possible. The reasons for this research program are as follows. First, it is desired to model a wide varicty of Trimpi obscrvations in order to confirm the theory. Secondly, accurate and reliable modelling of VLF scattering will enable VLF Trimpi data to be used to localise and map precipitation and possibly determine patch size and structure as well as the energy spectrum of precipitating electrons. Energetic electron precipitation from the magnetosphere into the ionosphere represents a substantial energy flux that is significant on a global scale. There is considerable interest in investigating this and, in particular, how it may be affected by global warming and by VLF radiation from industrial plant and power distribution systems.

It should be stated at this point that VLF lightning whistlers are not the only agent causing localised $D$ region anomalies that can scatter VLF radiation. High power VLF transmitters may cause patches, particularly if the transmissions trigger VLF emissions or chorus. In a fascinating series of experiments Barr et al. $(1984,1985)$ and Dowden et al. (1991) used a high power HF heater in Norway to create a transient localised $\mathrm{D}$ region patch with an elevated temperature and thus elevated collision frequency. The Trimpi phenomenon due to these patches was observed on short range paths. More recently, it has been postulated that both lightning strikes and high power terrestrial VLF transmitters may produce localised D region patches with elevated temperature, due to direct heating by VLF radiation. The appropriate observations will be found in Inan and Rodriguez (1993) and Inan (1990). This topic is still a matter of controversy, and it is not proposed to enter this debate in this paper. We will be content to note, though, that the theory and numerical modelling technique to be developed in this paper is easily modified to deal with patches of elevated collision frequency and also Trimpis on short paths as in Barr et al. $(1984,1985)$. Simulation of the Barr heater experiment will be the subject of a separate paper. In recent years it has been discovered that thunderclouds may discharge upwards to the ionosphere, giving rise to 'sprites' and 'blue jets'. This is an active area of research, well summarised in Strangeways (1996). Dowden et al. (1994) reported good correlation between Sprites and RORD's, which are early Trimpis with rapid onset and rapid decay. A Sprite will produce a transient vertical column of ionisation enhancement which will scatter VLF radiation in much the same way as a classical LIE. Again we note that the numerical techniques in this paper are eminently suitable for the modelling of RORD's.

\section{REVIEW OF THE OBSERVATIONAL LITERATURE}

There is a considerable body of literature covering observations of the VLF Trimpi effect. Space prohibits a thorough review of this excellent material. The reader will find useful reviews in Rycroft (1991, 1993), Strangeways (1996) and Smith (1996) which furnish comprehensive lists of references - at least up to those dates.

A number of institutions dominate in the literature on the Trimpi effect. These are Stanford University, Otago University in New Zealand, British Antarctic Survey, and the University of Natal at Durban. There are also important contributions from elsewhere, particularly Russia. A collection of papers representing a very useful data base are Wolf and Inan (1990), and Inan et al. $(1988 \mathrm{a}, \mathrm{b}, \mathrm{c})$. These papers deal with transmissions from NAA, NAU, NSS and NLK to Stanford and Lake Mistissini, in Canada. A further group of papers looks at Trimpis observed at Palmer and Siple, Antarctica, on long transmissions from various transmitters in North America. These papers are Carpenter and LaBelle (1982), Leyser et al. (1984), Carpenter et al. (1984, 1985), Inan et al. (1985), Hurren et al. (1986), Inan and Carpenter (1986, 1987). These papers establish the main observational features and also confirm VLF whistlers as the causative agent.

In 1990, Inan et al. (1990) first tackled the problem of the imaging of precipitation patches. Considerable progress on this problem was made by employing 
multiple criss-cross paths from 6 transmitters in the continental US to 3 receiver sites.

The University of Otago group in New Zealand has mainly used Trimpi observations on paths from NWC and Omega in Australia to an array of receiver sites in New Zealand. These are fairly short paths on which multimodal effects are expected to be prominent. The Otago data base is a valuable complement to that of Stanford, which is concerned with long oversea paths or intermediate length paths over land only. The paper by Dowden and Adams (1988) views precipitation patches as stalactites or localised regions, quite small with possible structure and sharp edges. The thrust of the Otago research has been that significant off-GLC path scattering may occur due to the fact that the patch is not a large object with a smooth Gaussian profile. This is supported by data in Dowden and Adams (1989a,b).

In 1990, the University of Otago group tackled the problem of patch localisation. Dowden and Adams (1990) and Adams and Dowden (1990) coined the concept of group delay $\tau$, using the 2 closely spaced frequencies of NWC transmissions. The path difference $s=c \tau$, where $\Delta f . \tau=\Delta \Theta$, which is the difference of scatter phase between the two frequencies. The measured group delay fixes the patch location (assumed single) on an cllipse. The Otago group have also set up a linear 'Golom' array of 4 receiver sites on S Island, New Zealand, with a further site at Wellington. By comparing the scatter phase at two receivers space $\sim \lambda$ apart the direction of the scattered signal may be inferred. This is very much akin to classical array beamforming. This arrival direction combined with the group delay ellipse will localise a single patch, at least in principle (see Dowden and Adams (1993a,b)).

British Antarctic Survey have assembled a useful data base of VLF Trimpis, mostly looking at paths from Siple, NAA, NSS and NPM to Halley Bay and Faraday stations in Antarctica. The reader is referred to Clark and Smith (1990), Smith and Cotton (1990), and Cotton and Smith (1991). In Smith et al. (1993) the first results from the OPAL 1989 campaign were presented and LIE location and size estimated from the Cotton/Smith model.

Further data has been presented by the University of Natal group in Durban. Friedel and Hughes (1990) surveyed Trimpis on 1982 Sanae tapes. Friedel and Hughes (1990, 1992) and Friedel et al. (1993) presented results for low latitude Trimpi events.

There is little doubt that the future thrust of research into classical Trimpis will be to collect data to enable patch localisation to be performed. Whatever technique is adopted for this 'inverse' problem, it will only be made possible by the simultaneous collection of data from multiple RX/TX pairs and at multiple frequencies. The utilisation of arrays of receivers, a technique pioneered by the Otago group, is probably the most systematic approach to the localisation problem.

\section{SUBIONOSPHERIC VLF PROPAGATION IN THE CASE OF A HOMOGENEOUS IONOSPHERE}

The problem of subionospheric VLF propagation between a horizontally homogeneous ionosphere and a homogeneous ground plane has been extensively studied in the literature. Since we are here dealing with propagation distances in the range $100-10000 \mathrm{~km}$, the modal theory of propagation represents the most accurate and appropriate means of tackling the propagation problem. Ray tracing techniques are neither helpful nor accurate at these ranges. Wait has been pivotal in the development of the modal theory of subionospheric VLF propagation; the reader is referred to the classic texts Wait (1970) and Budden (1961). Further information on VLF mode theory will be found in Wait (1970), Pappert and Bickel (1970), Pappert et al. (1967), Pappert and Hitney (1988), Pappert (1968, 1970), and Wait and Spies (1964).

For the purposes of this paper we need to remind ourselves of the basics of modal VLF propagation theory. MKS units will be used throughout this paper. Consider an oscillating vector electric dipole

$$
P_{d d}(t)=P_{0} \mathrm{e}^{j \omega t}
$$

located above the Earth's surface. Define a right handed coordinate system where $z$ is vertically upwards and $x$ is along the direction of propagation. Assuming that the source dipole is located at $\left(0,0, z_{s}\right)$, the complex vector electric field $E$ at the receiver at $\left(d, 0, z_{r}\right)$ is given by the matrix expression below, where it is assumed that the receiver is in the far field i.e. $d \gg \lambda$ (see Budden (1961), Poulsen et al. (1993a,b)).

$$
E=\left[\begin{array}{l}
E_{x} \\
E_{y} \\
E_{z}
\end{array}\right]=\frac{j \omega \tilde{c}}{\sqrt{d}} \sum^{m} \mathrm{e}^{-j k S_{0}^{n d}} F \Gamma_{m}\left(z_{S}\right) \Gamma_{m}\left(z_{\mathrm{R}}\right) \lambda^{m} \cdot P_{0}
$$

where

$$
P_{0}=\left[\begin{array}{l}
P_{x} \\
P_{y} \\
P_{z}
\end{array}\right] ; \quad k=\omega / c
$$

and

$$
\tilde{c}=-\frac{\mathrm{e}^{+j \pi / 4} \mu_{0} \omega}{h \sqrt{2 \pi k}}
$$


where $h$ is the reference height of the night-time ionosphere (see Wait (1962)). The quantity $F$ is a scalar spreading/focussing factor

$$
F=\sqrt{(d / R e) / \sin (d / R e)}
$$

where $R e$ is the Earth's radius. This factor is only significant for path lengths on a global scale, where the assumption of horizontal homogeneity becomes questionable anyway. The sum ' $m$ ' is over the allowed modes in the waveguide, $k S_{\mathrm{o}}^{m}$ being the complex horizontal wavenumber of mode $m$ at frequency $\omega$.

The tensor $\Gamma_{m}$ is defined as

$$
\Gamma_{m}=\left[\begin{array}{ccc}
\Gamma_{z}^{m}(z) & 0 & 0 \\
0 & \Gamma_{y}^{m}(z) & 0 \\
0 & 0 & \Gamma_{z}^{m}(z)
\end{array}\right]
$$

where $\Gamma_{x}^{m}, \Gamma_{y}^{m}$, and $\Gamma_{z}^{m}$ are the normalised complex height gain functions for fields $E_{x}, E_{y}$ and $E_{z}$ for mode number $m$. The matrix $\lambda^{m}$ is a matrix of complex excitation factors. The component $\lambda_{i j}^{m}$ is the excitation factor for electric field component $E_{i}$ by a dipole in the $j$ direction, where coordinates $x, y$ and $z$ correspond to indices 1, 2 and 3 in the matrix formulation. It should be noted that the curvature of the Earth is critical in calculating modal excitation factors and height gain functions.

In the scenarios to be modelled the transmitters are all vertical electric dipoles (ved's) at $z=0$. The ground receiver stations are either vertical electric dipoles or are assumed to be so. Some receivers may consist of crossed horizontal magnetic loops measuring $B_{x}$ and $B_{y}$, but where the direction of arrival of the scattered signal from the LIE is within a few degrees of the direct path, then $B_{y}$ and $E_{z}$ are directly related by

$$
E_{z} \cong j c B_{y}
$$

For scenarios with a scattering angle that is not negligible there is no direct relationship between $E$ and $B_{x}$ and $B_{y}$, and strictly speaking separate computations of observed Trimpis in $B_{y} / B_{x}$ would be necessary. Since the receivers are effectively regarded as being ved's we shall only consider vertical currents induced in the LIE, which act as the source for the scattered field.

In this paper, then, we shall only be concerned with vertical electric fields produced by ved's, and under these circumstances the propagation equation simplifies to

$$
E_{z}=\frac{j \omega \tilde{c} F}{\sqrt{d}} \sum_{m} \mathrm{e}^{-j k s_{0}^{m} d} \Gamma_{z}^{m}\left(z_{s}\right) \Gamma_{z}^{m}\left(z_{R}\right) \lambda_{33}^{m} P_{z}
$$

We note here that with excitation of the Earth-iono-

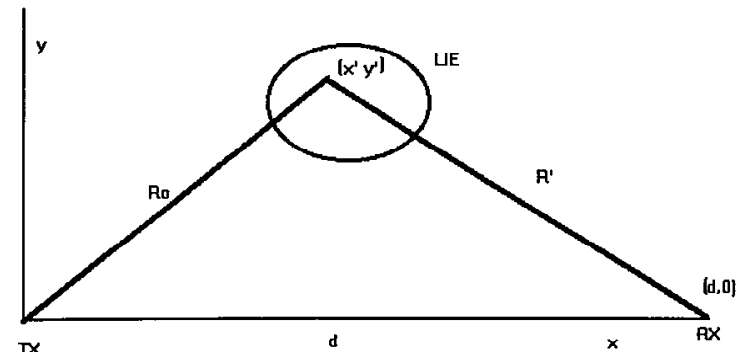

Fig. 2. Scenario for analysis of the Poulsen et al. (1993a, b) model, viewed in the horizontal $x, y$ plane.

sphere waveguide by vertical electric dipoles it is mainly the TM modes that are excited. For paths of order several thousand $\mathrm{km}$, it transpires that 2 or 3 dominant TM modes only are significant. For long oversea paths of order $10000 \mathrm{~km}$, only a single TM mode dominates.

\section{VLF SCATTERING BY IONOSPHERIC IRREGULARITIES - PREVIOUS WORK}

It is surprising how few researchers have analysed the problem of calculating quantitatively VLF scattering from $D$ region irregularities. Nearly all the work in this field has been done by Poulsen et al. (1990, 1993a,b). These papers base their approach on a theoretical expression due to Wait (see Wait $(1964 a, b))$. It is important for us to highlight the salient features of the Poulsen et al. approach.

Poulsen $e t$ al. consider a ved transmitter located at $(0,0,0)$. Figure 2 shows the overall scenario in the $x, y$ plane. The ionosphere is assumed homogeneous except in the LIE region, denoted $\wp$, where ionospheric parameters are perturbed. The vertical electric field at the receiver $E_{z}(d, 0,0)$ is expressed as a modal sum

$$
E_{z}=F \sum_{m} j \omega \tilde{c} \lambda_{33}^{m} \ell_{m}(d, 0)
$$

where the Wait (1970) expression has been rearranged a little. The variable $\ell_{m}(x, y)$ is the complex modal field amplitude for mode $m$. Under the assumption that the scale length of the ionospheric perturbation ' $a$ ' is large and satisfies $a \gg \lambda$, Wait asserted that intermodal coupling in $\wp$ would be negligible, and that the modal field $\ell_{m}$ satisfies the Helmholtz equation in any domain not including the source dipole.

$$
\left\{\frac{\partial^{2}}{\partial x^{2}}+\frac{\partial^{2}}{\partial y^{2}}+\left(k S^{m}\right)^{2}\right\} \ell_{m}=0
$$

where $k S^{m}$ is the localised parallel wave number for 
mode $m$ in the perturbed ionosphere, and $k S_{0}^{m}$ is the corresponding quantity for the unperturbed ionosphere. This result makes the entirely plausible assumption that the modes are weakly damped, i.e.

$$
\mathscr{I}\left(S^{m}\right) \ll 1
$$

Using a perturbation approach Wait (1970) showed that the modal signal $\ell_{m}$ at the receiver consists of a direct signal $\ell_{m}^{0}$ plus a signal $\ell_{m}^{s}$ scattered from the region $\wp$.

$$
\begin{gathered}
\ell_{m}(d, 0)=\ell_{m}^{0}(d, 0)+\ell_{m}^{s}(d, 0) \\
\ell_{m}^{s}=-\frac{j k_{0}^{2}}{4} \iint_{\xi}\left[S^{m^{2}}\left(x^{\prime}, y^{\prime}\right)-S_{0}^{m^{2}}\right] \ell\left(x^{\prime}, y^{\prime}\right) . \\
H_{0}^{(2)}\left(k S_{0}^{m} R^{\prime}\right) d x^{\prime} d y^{\prime}
\end{gathered}
$$

Wait (1970) now applies the Born, or weak, scattering approximation, replacing $\ell_{m}$ inside the integral by $\ell_{m}^{0}$, where in the homogeneous case

$$
\ell_{m}^{0}\left(x^{\prime}, y^{\prime}\right)=\frac{P_{z}}{\sqrt{R_{0}}} \mathrm{e}^{-j k S_{0}^{m} R_{0}}
$$

By using

$$
k S_{0}^{m} R^{\prime} \gg 1
$$

and substituting the asymptotic form of the Hankel function, he obtained an expression for the ratio of the scattered modal field $\ell_{m}^{s}$ to the direct modal field $\ell_{m}^{0}$.

$$
\begin{gathered}
\frac{\ell_{m}^{s}}{\ell_{m}^{0}}=-\frac{j k_{0}^{2}}{4} \sqrt{\frac{2 d j}{\pi k S_{0}^{m}}} \iint_{\xi} \frac{G\left(x^{\prime}, y^{\prime}\right) \mathrm{e}^{-j k S_{0}^{\prime \prime}\left(R_{0}+R^{\prime}-d\right)}}{\sqrt{R_{0} R^{\prime}}} d x^{\prime} d y^{\prime} \\
\tilde{G}\left(x^{\prime}, y^{\prime}\right)=\left\{S_{m}^{2}\left(x^{\prime}, y^{\prime}\right)-S_{0}^{m 2}\right\}
\end{gathered}
$$

Using only a single dominant TM mode Poulsen et al. (1990) used this expression to model VLF Trimpis observed on the path NPM (Hawaii)-Palmer, Antarctica, numerically. Good agreement with observations was obtained. In a subsequent paper, Poulsen et al. $(1993 \mathrm{a}, \mathrm{b})$ extended the model to encompass all allowable modes. Propagation along long paths was computed using the sophisticated LWPC code, which employs global ground conductivity maps and ionospheric maps, and calculates intermodal coupling continuously along propagation paths. However Wait (1970) unimodal scattering expression was used and there is no intermodal coupling due to the scattering process itself. Poulsen et al. (1993a,b) applied this new model to the path NSS-Stanford University. Good agreement with observations was obtained, and multimodal effects were extensively discussed.

Using equation (15), the scattering parameter is seen to be $\Delta\left(S^{2}\right)=2 S \Delta S \sim 2 \Delta S \sim 2 \Delta n$, where $n$ is the effective refractive index. Electron density enhancement in the $D$ region will lower the effective reflection height of the ionosphere, thus decreasing $S$. The refractive index $n$ is then less than unity and the patch will behave as a diverging lens. A LIE on the GLC path will then give a reduced VLF amplitude and a phase advance-the classic $(-+)$ Trimpi. Viewed mathematically we note that the quantity

$$
\eta^{\prime}=\angle \ell_{m}^{s}-\angle \ell_{m}^{0}
$$

gives the scattered signal phase minus the direct signal phase, for mode $m$. This we define as the 'scatter phase'. Since $\Delta(S)<0$ the Wait formula gives a scatter phase of 135 degrees for a small LIE located on the GLC path. This results in a classic $(-+)$ Trimpi. The critical reader will, however, point out that the concept of a point scatterer is inconsistent with the a priori assumption that $a \gg \lambda$.

We note again that the fundamental assumption underlying Wait (1970) formalism is that $a \gg \lambda$, and that inter-modal coupling at the LIE may be neglected. It is a fundamental axiom of this paper that this condition is not satisfied for a typical LIE. Indeed, dominant TM modes have values for $S_{0}$ within a few percent of one another, and it needs LIE scale sizes of order $1000 \mathrm{~km}$ in order to eliminate inter-modal scattering.

Wait (1980) considered the problem of the scattering of VLF waves by a small dielectric cylinder in a cylindrical waveguide geometry. The study was analytic, but Wait (1980) found substantial mode conversion as a result of the scattering process. This strong intermodal scattering is a very pronounced feature of the numerical model to be presented in this paper. In Wait (1995), this work has been continued and applied to VLF scattering from a column of ionization in the Earth-ionosphere waveguide. Wait $(1991,1995)$ found significant mode conversion where the ionization is limited in height or a function of height. This conclusion is consistent with the theory in this paper. Another recent important paper is by Baba and Hayakawa (1995). This paper uses the finite element method, and, like the present paper, includes scattering into other modes by the LIE. It also permits scattcring from LIE patches with strong gradients to be modelled accurately. Baba and Hayakawa (1995) give a $2 \mathrm{D}$ representation of the problem, which is otherwise rigorous and which does not employ a Born/weak scattering approximation. When extended to $3 \mathrm{D}$, which it will be eventually, the method promises to be an exceptionally powerful modelling tool. Currently their approach nicely complements that of this paper. The approach here is ' $3 \mathrm{D}$ and Born', theirs '2D and non Born'. 
Nickolaenko (1994) investigated ELF propagation in the Earth-ionosphere waveguide in the presence of inhomogeneities in the ionospheric reflection height and in the surface reflection characteristics of the lower ionospheric boundary.

\section{DESCRIPTION OF THE MODELLING METHOD}

The theory and modelling of VLF scattering to be developed in this paper is straightforward in concept. Before developing the mathematical formalism we shall describe the essential features of the method.

(a) The formalism is one of point scattering. The scattered wavefield from a single infinitessimal element $\mathrm{d} \tau$ of the LIE will be calculated. The LIE is viewed as a three dimensional assembly of point scatterers and the total scattered field is found from an integral over the volume of the LIE. The LIE can have any distribution of electron density/collision frequency in space, and it is not required that the scale length of the LIE in the horizontal plane be many wavelengths long. The method actually works better for small scattering volumes, which place less strain on the Born weak scattering approximation.

(b) The method naturally uses all the allowed modes in the Earth ionosphere waveguide, both TM and TE, and is thus suitable for scattering calculations for quite short paths. The incident field $E_{0}$ at an element $\mathrm{d} \tau$ of the LIE is first calculated using VLF modal propagation theory. The Born weak scattering approximation is involved by calculating $E_{0}$ under the assumption of a homogeneous ionosphere. The equivalent induced dipole source $J_{\text {eff }} \mathrm{d} \tau$ is calculated, which is a function of the susceptibility tensor and its perturbation in the LIE. The elevated source dipole $J_{\text {eff }} \cdot \mathrm{d} \tau$ reradiates the scattered field, which is again calculated using VLF modal propagation theory. The final total scattered field is obtained by integrating over the volume of the LIE.

(c) This approach to the scattering problem immediately predicts substantial intermodal scattering. The field $E_{0}$ incident at an element of the LIE consists of a modal sum. The source dipole $J_{\text {eff }} \mathrm{d} \tau$ will reradiate into all modes. Only when the LIE size is very large $(>1000 \mathrm{~km})$ will the intermodal scattering terms be eliminated in the integration over LIE volume.

\section{THE SCATTERING EQUATIONS}

We assume that all variables have a time dependence as $\exp (j \omega t)$. Assuming a magnetic susceptibility of unity, we may apply Maxwell's equations to the Earth-ionosphere waveguide system to give

$$
\begin{gathered}
\nabla \times E=-j \omega \mu_{0} H \\
\nabla \times B=\mu_{0} j+j \omega \mu_{0} \varepsilon_{0} E
\end{gathered}
$$

where all fields are complex vectors and functions of position vector $r$. The latter equation may be written

$$
\nabla \times H=J_{s}+j \omega P+j \omega \varepsilon_{v} E
$$

where $J_{s}(r)$ is the source current of the transmitter, and $P$ is the polarisation vector in either the ionosphere or the ground. For a linear medium this vector may be written

$$
P=\varepsilon_{0} \chi E=J / j \omega=\sigma E / j \omega
$$

where $\chi(r)$ is the complex susceptibility tensor and $I$ is the current density induced in the medium. Eliminating $H$ between equations (17) and (19) we obtain

$$
\nabla \times(\nabla \times E)+j \omega \mu_{0}\left[J_{s}+j \omega P+j \omega \varepsilon_{0} E\right]=0
$$

which becomes

$$
\nabla \times(\nabla \times E)-\frac{\omega^{2}}{c^{2}}(1+\chi) E=-j \omega \mu_{0} J_{s}(r)
$$

A rigorous solution of the VLF scattering problem requires the global solution of this equation in the domain $\{r\}$ covering the lower ionosphere, subionospheric waveguide and ground. Since we have a localised ionospheric inhomogeneity we may express the susceptibility tensor as

$$
\chi(r)=\chi_{0}(r)+\chi^{\prime}(r)
$$

where $\chi_{0}(r)$ is the zero order susceptibility and $\chi^{\prime}(r)$ is the perturbation of $\chi$, assumed nonzero in a localised region (the LIE) located in the $D$ region of the ionosphere. Similarly we may write

$$
E(r)=E_{0}(r)+E^{\prime}(r)
$$

where $E_{0}(r)$ is the radiation ficld of the transmitter in the homogeneous case and $E^{\prime}(r)$ is the scattered wavefield. Substituting equations (23) and (24) into equation (22) we obtain

$$
\nabla \times\left(\nabla \times E_{0}\right)-\frac{\omega^{2}}{c^{2}}\left(1+\chi_{0}\right) E_{0}=-j \omega \mu_{0} J_{\mathrm{s}}(r)
$$

whose solution $E_{0}$ is that of the homogeneous problem, and also

$$
\nabla \times\left(\nabla \times E^{\prime}\right)-\frac{\omega^{2}}{c^{2}}\left(1+\chi_{0}\right) E^{\prime}-\frac{\omega^{2}}{c^{2}} \chi^{\prime}\left(E_{0}+E^{\prime}\right)
$$

The latter expression may be rearranged as follows

$$
\nabla \times\left(\nabla \times E^{\prime}\right)-k^{2}\left(1+\chi_{0}\right) E^{\prime}=-j \omega \mu_{0} J_{\mathrm{err}}(r)
$$

where 


$$
J_{\mathrm{eff}}(r)=\frac{j k^{2}}{\omega \mu_{0}} \chi^{\prime}(r)\left\{E_{0}(r)+E^{\prime}(r)\right\}
$$

We see that equation (27) is of the same form as equation (25), and thus the scattered field $E^{\prime}(r)$ is sourced on the current field $J_{\text {eff }}(r)$, which may be regarded as the current field 'induced' in the LIE. The self consistent solution of equations (25) and (28) is virtually an impossible task. We now apply the Born weak scattering approximation and put $\chi^{\prime}(r) \ll \chi_{0}(r)$, and so put $E^{\prime} \rightarrow 0$ in the expression for $J_{\text {eff }}$, whence

$$
J_{\text {eff }}(r) \cong \frac{j k^{2}}{\omega \mu_{0}} \chi^{\prime}(r) E_{0}(r)
$$

In the present problem the Born approximation is a reasonable one, essentially requiring that the WKB phase at the centre of the LIE is not perturbed by more than about 15 degrees, and that the locally computed height gain functions in the LIE do not differ from those in the homogeneous case by more than about $20 \%$ at relevant altitudes. In the case of very large or very 'strong' LIE's, the Born approximation will begin to fail, but techniques are available to tackle such situations.

We have assumed that $E^{\prime}=0$ in the scattering volume, but other variants of the Born approximation are available and are to be preferred. The typical LIE will appear as a disc some $100 \mathrm{~km}$ in horizontal extent and maybe about $20 \mathrm{~km}$ thick. This disc sees an externally applied vertical electric field. By analogy with the problem of a field applied to an isotropic dielectric slab, the approximation $D^{\prime}=0$ would be preferable since this would take into account polarisation of the slab. Putting $D^{\prime} \rightarrow 0$ in the LIE we now get

$$
D_{0}=\left(1+\chi_{0}\right) E_{0}=D=\left(1+\chi_{0}+\chi^{\prime}\right)\left(E_{0}+E^{\prime}\right)
$$

whence

$$
E(r)=\left(1+\chi_{0}+\chi^{\prime}\right)^{-1}\left(1+\chi_{0}\right) E_{0}
$$

The effective source current $J_{\text {eff }}$ is now given by

$$
J_{\text {efr }}(r)=\frac{j k^{2} \chi^{\prime}}{\omega \mu_{0}}\left(1+\chi_{0}+\chi^{\prime}\right)^{-1}\left(1+\chi_{0}\right) E_{0}
$$

A more general expression is a linear interpolation between equations (29) and (32), where $\alpha$ is an adjustable parameter dependent on LIE geometry:

$$
J_{\mathrm{err}}(r)=\frac{j k^{2}}{\omega \mu_{0}} \chi^{\prime}\left[1+\alpha \chi+(1-\alpha) \chi_{0}\right]^{-1}\left(1+\chi_{0}\right) E_{0}
$$

For a smaller LIE the geometry will be closer to a sphere. Now in the case of an isotropic dielectric sphere, susceptibility $\chi_{0}+\chi^{\prime}$, embedded in a medium of susceptibility $\chi_{0}$, and subjected to an external field $E_{0}$, the field in the sphere is given by

$$
E=\left(1+\frac{1}{3} \chi+\frac{2}{3} \chi_{0}\right)^{-1}\left(1+\chi_{0}\right) E_{0}
$$

which points to a choice of $\alpha=1 / 3$. In the simulations to follow we shall use $\alpha=2 / 3$ throughout. Note that all these expressions for $J_{\text {eff }}$ coalesce in the Born limit, and the choice of $\alpha$ in practice only has a slight effect on the computed results.

\section{CALCULATION OF THE SUSCEPTIBILITY TENSOR $\chi$}

This is a standard analysis and we shall here briefly derive an appropriate expression for $\chi(r)$, assuming all fields have a time dependence as $\exp (j \omega t)$. In the ionosphere we assume a cold electron plasma, density $N_{e}(z)$, collision time $\tau(z)$. Ion motion is ignored and electron motion is assumed linear. We take a localised set of rectilinear coordinates with $z$ assumed vertically upwards. The ambient magnetic field $B_{0}(z)=\left[B_{x}, B_{y}\right.$, $B_{\mathrm{z}}$ ]. The linear equation of motion of the electrons at height $z$ is given by

$$
m \dot{v}=-e E-m v / \tau-e\left(v \times B_{0}\right)
$$

and the corresponding plasma current is given by

$$
J=-e N_{e} v
$$

whence

$$
P-\epsilon_{0} \chi E=J / j \omega=-e N_{\mathrm{e}} v / j \omega
$$

Defining

$$
m^{*}=m \xi=m(1+1 / j \omega \tau)
$$

the equation of motion becomes

$$
j \omega m^{*} v=-e E-e\left(v \times B_{0}\right)
$$

This may be written in the form

$$
j \omega Q \cdot v=-\frac{e}{m^{*}} E
$$

where

$$
Q=\left[\begin{array}{ccc}
1 & \frac{-j \Omega_{z}}{\omega \xi} & \frac{j \Omega_{y}}{\omega \xi} \\
\frac{j \Omega_{z}}{\omega \xi} & 1 & \frac{-j \Omega_{x}}{\omega \xi} \\
\frac{-j \Omega_{y}}{\omega \xi} & \frac{j \Omega_{x}}{\omega \xi} & 1
\end{array}\right]
$$

and $\Omega_{x}=e B_{x} / m$ etc. We may now write 


$$
P=-\frac{e N_{e}}{j \omega}\left(\frac{-e}{j \omega m^{*}}\right) Q^{-1} E=-\frac{\Pi_{\varepsilon}^{2} \epsilon_{0}}{\omega^{2} \xi} Q^{-1} E
$$

which gives the following expression for susceptibility

$$
\chi=-\frac{\Pi_{e}^{2}}{\omega^{2} \xi} Q^{-1}
$$

where all variables except $\omega$ are functions of $z$. The quantity $\Pi_{e}$ is, as usual, the electron plasma frequency.

$$
\Pi_{e}^{2}=N_{e} e^{2} / \epsilon_{0} m
$$

The unperturbed ionospheric susceptibility $\chi_{0}$ is given by

$$
\chi_{0}(z)=\frac{-\Pi_{e}^{0^{2}}}{\omega^{2}(1+1 / j \omega \tau)} Q_{0}^{-1}
$$

and the perturbation of susceptibility $\chi^{\prime}$ by

$$
\chi^{\prime}(z)=\frac{-1}{\omega^{2}} \Delta\left\{\frac{\Pi_{e}^{2} Q^{-1}}{(1+1 / j \omega \tau)}\right\} .
$$

where the perturbation of the ionospheric plasma is only in density, the $Q$ matrix is constant and we may write

$$
\chi^{\prime}=\chi_{0} \Delta N_{e} / N_{e}
$$

In the case of a LIE produced by heating, $\tau$ is perturbed and the full complex expression for $\chi^{\prime}$ will need to be computed.

\section{FORMAL SOLUTION OF THE VLF SCATTERING EQUATIONS USING THE VLF MODE THEORY OF PROPAGATION}

We now develop solutions to the scattering equations using VLF modal propagation theory. This requires that the modal sum provides an accurate description of the field, which holds for path lengths greater than about $10 \mathrm{~km}$. We consider the classic Trimpi scenario illustrated in Fig. 2. A right hand set of orthogonal coordinates is used with $x$ along the transmitter (TX)-Receiver(RX) axis and $z$ vertically upwards. The transmitter is a vertical electric dipole of moment

$$
P_{z} \mathrm{e}^{j \omega t}
$$

located at $(0,0,0)$. The receiver is located at $(d, 0,0)$, and is also a ved antenna. The ionosphere is homogeneous except in the region of the LIE. We consider an elementary scattering volume in the LIE, $d \tau=d x^{\prime} d y^{\prime} d z^{\prime}$ located at $r^{\prime}=\left(x^{\prime}, y^{\prime}, z^{\prime}\right)$. The distances $R_{0}$ and $R^{\prime}$ are horizontal ranges

$$
R_{0}=\sqrt{x^{\prime 2}+y^{\prime 2}} ; \quad R^{\prime}=\sqrt{\left(d-x^{\prime}\right)^{2}+y^{\prime 2}}
$$

The direct Great Circle (GC) path signal $E_{z}^{0}$ observed at $\mathrm{RX}$ is given by equation 2

$$
E_{z}^{0}=\frac{\tilde{c}}{\sqrt{d}} \sum_{m} \mathrm{e}^{-j k S_{0}^{n} d} \lambda_{33}^{m}(j \omega) P_{z} F(d)
$$

where

$$
\tilde{c}=\frac{\mu_{0} \omega}{h \sqrt{2 \pi k}} \mathrm{e}^{j 225^{0}} ; \quad F(u)=(u / R e) /(\sin (u / R e))
$$

At this point we make an entirely reasonable assumption. The height gain functions and excitation factors have a weak dependence upon the direction of propagation relative to magnetic North. In the Trimpi scenario all paths will be within, say, 10 degrees of the GC direct path direction TX-RX, so we use throughout the set $\lambda_{i j}^{m}$, etc. appropriate to that direction. Now the vector field $E_{0}\left(x^{\prime}, y^{\prime}, z^{\prime}\right)$ incident on the LIE element is, invoking Born and assuming homogeneity,

$$
E_{0}=\frac{\tau}{\sqrt{R_{0}}} \sum_{m} \mathrm{e}^{-j k S_{0}^{m} R_{0}} \Gamma_{m}\left(z^{\prime}\right) \lambda^{m} p(j \omega) F\left(R_{0}\right)
$$

and $p^{T}=\left(0,0, P_{z}\right)$. The induced vector source current $J_{\text {eff }}$ may be written

$$
J_{\text {eff }}=\tilde{\sigma} E_{0}
$$

where we have introduced an effective conductivity matrix $\tilde{\sigma}$ given by

$$
\tilde{\sigma}=\frac{j k^{2}}{\omega \mu_{0}} \chi^{\prime}\left(1+\frac{2}{3} \chi+\frac{1}{3} \chi_{0}\right)^{\prime}\left(1+\chi_{0}\right)
$$

The susceptibility perturbation field $\chi^{\prime}\left(r^{\prime}\right)$ describes the ionospheric inhomogeneity and $\chi_{0}\left(z^{\prime}\right)$ is the unperturbed susceptibility. The scattered field $E^{s}$ observed at RX is sourced on field $J_{\text {eff }}\left(r^{\prime}\right)$. Applying modal theory again (equation 2) we get

$$
E^{s}=\tilde{c} \iiint_{\vartheta} \sum_{m^{\prime}} \frac{\mathrm{e}^{-j k S_{0}^{m^{\prime}} R^{\prime}}}{\sqrt{R^{\prime}}} F\left(R^{\prime}\right) \Gamma_{m^{\prime}} \lambda^{m^{\prime}} \tilde{\sigma}\left(r^{\prime}\right) E_{0} d \tau
$$

where the integral is over the $3 \mathrm{D}$ volume of the LIE. Substituting for $E_{0}$ we now secure

$$
\begin{gathered}
E_{z}^{s}=\tilde{c}^{2} \sum_{m^{\prime}} \sum_{m} \iiint_{\psi^{\prime}}\left\{\frac{\mathrm{e}^{-j k S_{0}^{m} R_{0}} \mathrm{e}^{-j k S_{0}^{m^{\prime}} R^{\prime}} F\left(R_{0}\right) F\left(R^{\prime}\right)}{\sqrt{R_{0} R^{\prime}}}\right\} \\
\cdot \hat{z} \Gamma_{m}\left(z^{\prime}\right) \lambda^{m^{\prime}} \tilde{\sigma}\left(r^{\prime}\right) \Gamma_{m}\left(z^{\prime}\right) \lambda^{m} p(j \omega) d \tau \\
\hat{z}=(001)
\end{gathered}
$$

The above equation represents a formal solution to the VLF scattering problem, and may be computed in a straightforward way. Subject to the Born approxi- 
mation and the validity of the VLF modal expansion, this expression is rigorous.

We now make a further approximation and ignore all elements of $\tilde{\sigma}$ except $\tilde{\sigma}_{33}$. This is equivalent to assuming that:

(a) The electric field $E_{0}$ incident on the LIE is vertical, since the TX is a ved. Pappert and Hitney (1988) give explicit numerical calculations which show that the vertical component of field is generally much larger than the horizontal.

(b) The horizontal currents induced in the LIE (not necessarily small) give negligible vertical fields at RX. These are good assumptions but not perfect. Work is currently underway at Southampton University by $\mathbf{R}$. Yeo on the modelling of the Trimpi effect using the whole $\tilde{\sigma}$ matrix. Assuming that $\tilde{\sigma}_{33}$ is the only non zero element, we obtain

$$
\begin{array}{r}
E_{z}^{\mathrm{v}}=\vec{c}^{\prime} \sum_{m^{\prime}} \sum_{m} \iiint_{\xi^{\prime}}\left\{\frac{\mathrm{e}^{-j k S_{0}^{n} R_{0}} \mathrm{e}^{-j k S_{01}^{n^{\prime}}} R^{\prime} F\left(R_{0}\right) F\left(R^{\prime}\right)}{\sqrt{R_{0} R^{\prime}}}\right\} \\
\cdot \Gamma_{z}^{m^{\prime}}\left(z^{\prime}\right) \Gamma_{z}^{m}\left(z^{\prime}\right) \tilde{\sigma}_{33}\left(r^{\prime}\right) \lambda_{33}^{m} \lambda_{33}^{m^{\prime}} P_{z}(j \omega) d \tau \\
=\tilde{c}^{2} \sum_{m^{\prime}} \sum_{m} \iiint_{\xi j}\{\cdots\} \lambda_{33}^{m} \eta^{m m^{\prime}}\left(r^{\prime}\right) d \tau P_{z}(j \omega)
\end{array}
$$

The terms in curly brackets are propagation terms. The square matrix $\eta^{m m^{\prime}}$ is a function of position $r^{\prime}$ in the LIE and represents a modal scattering matrix

$$
\eta^{m m^{\prime}}\left(r^{\prime}\right)=\lambda_{33}^{m^{\prime}} \tilde{\sigma}_{33}\left(r^{\prime}\right) \Gamma_{z}^{m}\left(z^{\prime}\right) \Gamma_{z}^{m^{\prime}}\left(z^{\prime}\right)
$$

The element $\eta^{m m}$ indicates how a point scatterer transfers energy from mode $m$ to mode $m^{\prime}$. A cursory inspection reveals that off diagonal elements will be of the same order as diagonal elements. We may also write

$$
E_{z}^{s}=\tilde{c}^{2} \sum_{m} \sum_{m} \iint_{\xi^{\prime}}\{\cdots\} \bar{\eta}^{m m^{\prime}}\left(x^{\prime}, y^{\prime}\right) \lambda_{33}^{m}(j \omega) P_{z} d x^{\prime} d y^{\prime}
$$

where

$$
\bar{\eta}^{m m^{\prime}}\left(x^{\prime}, y^{\prime}\right)=\int \eta^{\mathrm{mm}}\left(r^{\prime}\right) d z^{\prime}
$$

is the modal scattering matrix for a LIE highly localised in the $x^{\prime}, y^{\prime}$ plane. This matrix is quite sensitive to the function $\tilde{\sigma}_{33}\left(z^{\prime}\right)$ and thus to the vertical profile $\Delta N_{e}\left(z^{\prime}\right)$. Again it will be found that $\bar{\eta}$ has off diagonal elements of the same order as the diagonal elements. Further integration over $x^{\prime}, y^{\prime}$ will give the scatter matrix for the entire LIE. This further integration will scarcely affect the level of intermodal scattering between dominant TM modes, for which $S_{0}^{7}$ are within $1 \%$ of each other. Thus the matrix $\bar{\eta}$ is a good indicator of intermodal scattering. It should be emphasised that the size, structure and edge sharpness of the
LIE have little effect upon inter-modal conversion, because the horizontal wave numbers of dominant modes are so close. In order for the signals scattered into other modes to be dephased on integrating over the LIE, the LIE must be very large indeed - of order of several $1000 \mathrm{~km}$.

The ved TX and also the induced vertical currents in the LIE excite mainly 1-4 dominant TM modes. Significant scattering between dominant TM modes will occur. This cannot fail to be very important. Scattering into higher order TM modes that are strongly damped may be significant, particularly if the LIE is close to either the transmitter or the receiver.

As in the theory of Wait (1970), employed by Poulsen (Poulsen et al., 1993a,b), we define the ratio of scattered field to direct field

$$
\Theta=E_{z}^{s} / E_{z}^{0}
$$

where $\eta=\arg (\Theta)$ is the scatter phase (i.e. the phase of the scattered signal relative to that of the direct signal). The phase Trimpi $T_{\phi}$ in degrees is given by

$$
T_{\phi}=\arg \left\{E_{z}^{0}+E_{z}^{s}\right\}-\arg \left\{E_{z}^{0}\right\}
$$

and the magnitude Trimpi $T_{m}$ by

$$
T_{m}=20 \log _{10}\left\{\left|E_{z}^{0}+E_{z}^{s} / /\right| E_{z}^{0} \mid\right\}
$$

\section{Some comments on scatter phase}

It is of considerable interest and importance to examine the scatter phase $\eta^{\prime}$ produced by a point scatterer on the GC path. If we only consider one dominant TM mode $m$ ( $m=2$ or 3, depending on range) and ignore mode coupling effects we see that

$$
\eta^{\prime}=\angle \lambda_{33}^{m}+\angle \tilde{\sigma}_{33}^{m} /\left(z^{\prime}\right)+\angle \tilde{c}+2 \angle \Gamma_{z}^{m}\left(z^{\prime}\right)
$$

At low altitudes ( $60 \mathrm{~km}$ say) $\tilde{\sigma}_{33}$ is found to be almost entirely resistive and thus real positive. The excitation factor $\lambda_{33}^{m}$ is almost real positive, and the height gain factor squared will be close to real positive. Under these circumstances, a scatter phase of 225 degrees is predicted. Thus a low altitude resistive point scatterer on the GLC path will give a (--) Trimpi. Consideration of the problem of a plane wave incident normally on a strip perturbation reveals that this phase of 225 degrees must be correct. If the strip is dissipative, with a positive real part for $\tilde{\sigma}_{33}$, this is the only phase which will give an overall decrease of wave energy. In practice the LIE must have finite extent which will give a further phase lag. The perturbation normally extends to higher altitudes where further phase lags are acquired from the conductivity and from the height gain function squared. For a typical long range unimodal Trimpi problem of the kind 
examined by Poulsen et al. $(1993 \mathrm{a}, \mathrm{b})$ the scatter phase usually ends up in the 90-180 degree quadrant. The theory presented in this paper does have significant differences from that of Poulsen et al. (1993a,b). In the latter case, in the Born limit, the scatter phase of a point scatterer of any vertical structure on the GC path will always be exactly 135 degrees. This observation is qualified by the fact that strictly speaking a point scatterer violates the a priori assumption of Poulsen et al. (1993a,b) that $a \gg \lambda$. In the case of the theoretical development in this paper, in the Born limit the scatter phase of a point scatterer on the GLC path is a function of perturbation profile $\Delta N_{r}(z)$, and will thus be a function of time in general. The scatter phase steadily decreases with altitude, varying from 225 degrees for low altitude scatterers, and falling into the 90-180 degree quadrant at altitudes $>75 \mathrm{~km}$ approximately.

\section{COMPUTED RESULTS}

We shall now proceed to model numerically the VLF Trimpi effect on three paths, for which there is an abundance of data available. For reasons of space we shall not attempt detailed comparisons with individual events or groups of events. Such exercises will be the subject of further papers and will use more advanced versions of the code now under development. Also for reasons of space, we shall not attempt here a detailed modal breakdown of the results. The paths chosen for analysis are (i) NPM Hawaii-Palmer, Antarctica, (ii) NWC (Australia)Dunedin N.Z., and (iii) Omega(Australia)-Dunedin, N.Z.

The numerical modelling code essentially evaluates equation 54 for each scenario. The integration over the volume of the patch is replaced by a summation over a 3D grid. Here we employ a grid of $40 \times 40 \times 40$ which is more than adequate. The efficacy of the grid was tested in experimental runs with very fine grids. Discretisation of the patch volume does not affect inter-modal coupling at all, but can give spurious grating lobes at large scattering angles if the mesh is too coarse. The height gain functions $\Gamma_{z}(z)$ for the $E_{z}$ field, the excitation factors $\lambda_{33}$ and parallel wave numbers $k S_{0}^{m}$ are computed for all allowable TM and TE modes $m$, using the NOSC MODEFNDR package (Morfitt and Shellman, 1976). The reader will find much useful material relevant to MODEFNDR in the Proceedings of the CCIR XVIIth Plenary Assembly, Dusseldorf, 1990 though this document may be difficult to obtain. MODEFNDR uses a model for the electron density profile $N_{t}(z)$ as follows

$$
N_{v}(z)=\left(7.857 \cdot 10^{-5}\right) \mathrm{e}^{\beta\left(z-h^{\prime}\right.} v(z)
$$

where height $z$ and $h^{\prime}$ are in $\mathrm{km}$, and the electron density $N_{e}(z)$ is in electrons $\mathrm{cm}^{-3}$. The collision frequency $v(z)$ is assumed to have an exponential dependence on $z$ as follows

$$
v(z)=1 / \tau(z)=1.86 \cdot 10^{11} \mathrm{e}^{-0.15 z} \mathrm{~s}^{-1}
$$

MODEFNDR is invoked at a point midway along the TX-RX path, and the appropriate value of ambient field $B_{0}$ and dip angle are input. Suitable night time values of the ionospheric parameters $h^{\prime}$ and $\beta$ are also selected. All modal parameters are computed for a direction of propagation along the TX-RX axis. Two major disadvantages with MODEFNDR must be pointed out straightaway. First, this model assumes a homogeneous ionosphere, apart from the LIE. For long paths and for mixed paths over land and water this is hardly satisfactory. Poulsen et al. (1993a,b) use the LWPC software to calculate propagation along long paths. LWPC calculates modal VLF propagation over an inhomogeneous globe and provides a WKB solution with mode conversion along each inhomogeneous path. The next version of this software, under development by R Yeo, will also use LWPC. A second problem concerns both MODEFNDR and LWPC. Pappert and Hitney (1988) showed that the height gain functions become very small above about $85 \mathrm{~km}$. Little scattering will therefore occur from altitudes above this. However, the height gain functions returned by LWPC and MODEFNDR are inaccurate at altitudes $>90 \mathrm{~km}$. and do not become small. The computations in this paper will therefore only extend to $85 \mathrm{~km}$ as contributions from higher altitudes will be inaccurate, and almost certainly overestimated. At some stage Trimpi modellers will have either to upgrade or rewrite LWPC in order to correct this limitation.

\section{Path NPM Hawaii-Palmer Antarctica}

The first path to be modelled is NPM Hawaii to Palmer, Antarctica, on a single frequency of 23400 $\mathrm{Hz}$. The geographic path is shown in Fig. 3. This is an over water path of $12300 \mathrm{~km}$, and propagation will be dominated by the 1 TM mode over these distances. Ionospheric parameters $\beta=0.43$ and $h^{\prime}=87 \mathrm{~km}$ are selected. The 'ground' conductivity $\sigma=0.2 \mathrm{~S}$ and dielectric constant $t=81$ are values appropriate for seawater. The LIE is assumed elliptical in shape, orientated magnetic $\mathrm{E} \mathrm{W}$, and centred at $X_{c}, Y_{c} . \Lambda$ moderate sized LIE is assumed with major axis a $=300 \mathrm{~km}$ and minor axis $b=100 \mathrm{~km}$. The assumed vertical profile of unperturbed electron density $N_{e}(z)$ and total perturbed electron density $N_{e}(z)+\Delta N_{e}(z)$, at 


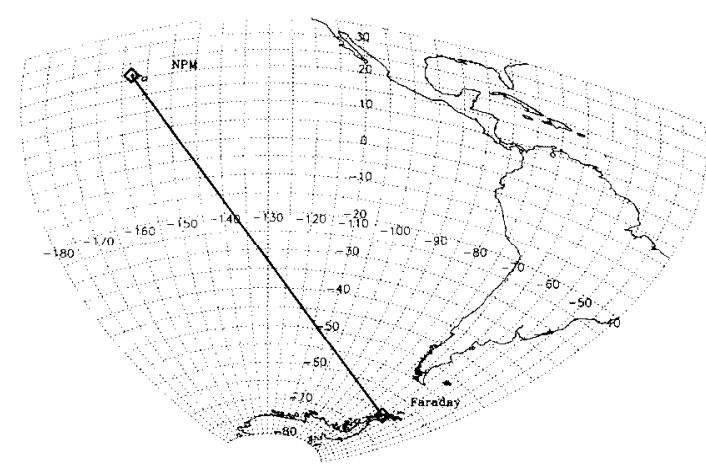

Fig. 3. Map of path NPM Hawaii-Palmer, Antarctica, which is near Faraday, Antarctica.

the LIE centre $X_{c}, Y_{c}$, are shown in Fig. 4. These values were selected after consultation of the paper by Wolf and Inan (1990). The profile is cutoff at $84 \mathrm{~km}$ to prevent inaccurate computations of scattering from very high altitudes. The profile $\Delta N_{e}(z)$ is shaded over the region of the LIE by a function $F_{s}(x, y)$ as follows

$$
\begin{gathered}
\Delta N_{e}(x, y, z)=\Delta N_{e}(z) F_{s}(x, y) \\
F_{s}=1 /\left(1+\xi^{10}\right) \\
\xi=\left(\frac{x-x_{c}}{a}\right)^{2}+\left(\frac{y-y_{c}}{b}\right)^{2}
\end{gathered}
$$

This is a Butterworth type dependence, giving a nearly constant precipitation strength over the ellipse,

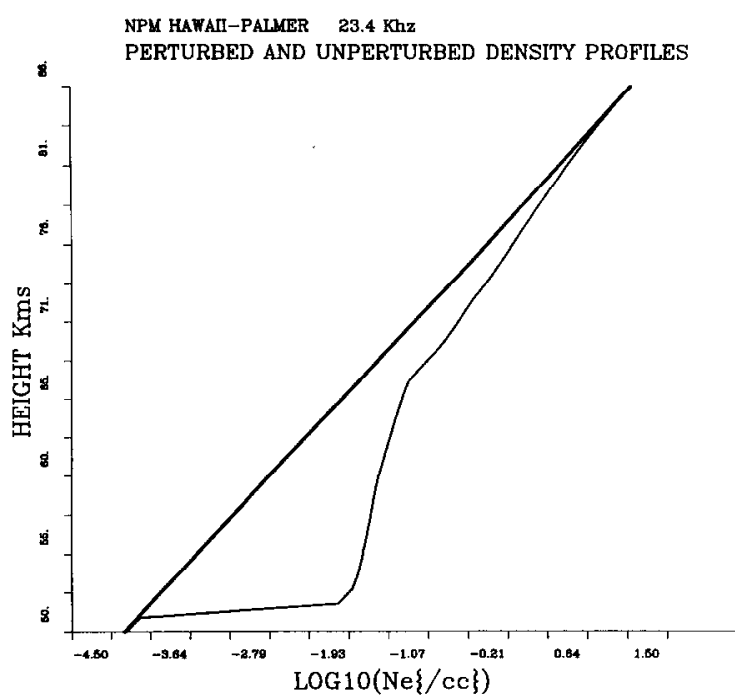

Fig. 4. Vertical profile of perturbed electron density $N_{e}(z)+$ $\Delta N_{e}(z)$ and unperturbed electron density $N_{o}(z)$ assumed for Trimpi calculations on the path NPM Hawaii-Palmer, presented as $\log$ to base 10 of density of electrons $\mathrm{cm}^{-3}$.

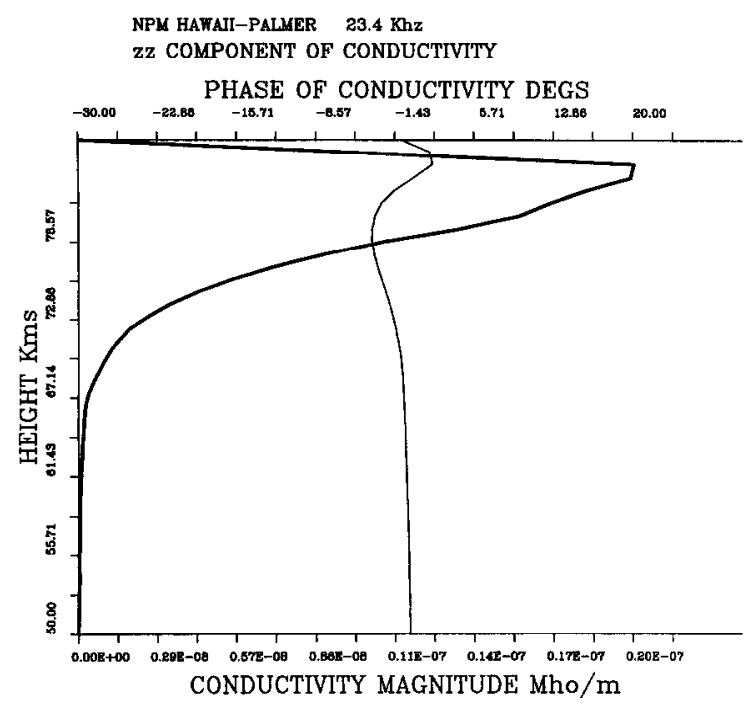

Fig. 5. Path NPM Hawaii Palmer. Plot of magnitude (heavy curve) and phase of vertical conductivity $\tilde{\sigma}_{33}$ as a function of height $z$, measured at the centre of the LIE.

with a rapid falloff at the boundary. The code has the facility to insert horizontal fine structure into the function $\Delta N_{e}(x, y, z)$, but we have not utilised this in the absence of any hard observational data as to what form such fine structure might take. Some further experimental runs were done with a low order (2) Butterworth dependence, giving a 'soft' edge to the LIE. This had little effect on intermodal scattering, but did give a narrower beamwidth and less off-axis scattering.

Next we present some of the parameters that go to determine the Trimpi phase and amplitude perturbations. Figure 5 shows the vertical distribution of $\tilde{\sigma}_{33}(z)$ at the centre of the LIE. This equivalent vertical conductivity is seen to be close to real, except at high altitudes where there is a few degrees of phase lag. The ionosphere appears resistive and the equivalent source current $J_{\text {eff }}$ is nearly in phase with the incident field $E_{0}$. Conductivity magnitude increases roughly exponentially with height, due to the exponential dependence of collision frequency on $z$. Of course $\tilde{\sigma}_{33}$ falls to zero at the top of the height range as $\Delta N_{e} \rightarrow 0$.

It is of considerable interest to examine the modal scattering matrix $\eta$, evaluated at the centre of the LIE as a function of $z$. Figure 6 plots the magnitudes of three diagonal elements $\eta^{m m}$ for the three dominant TM modes, which MODEFNDR labels $m=3,2$ and 5 . The magnitude of scattering from mode 3 to mode 3 is maximum at about $75 \mathrm{~km}$, and there is significant scattering over the height range $70-80 \mathrm{~km}$. The scattering of the next most important mode $m=2$, the so 
NPM HAFAII-PALMER $23.4 \mathrm{kHz}$

DIAGONAL COUHLING CUEF'FICIE'NT'S 3 DOMINANT TM MUDES

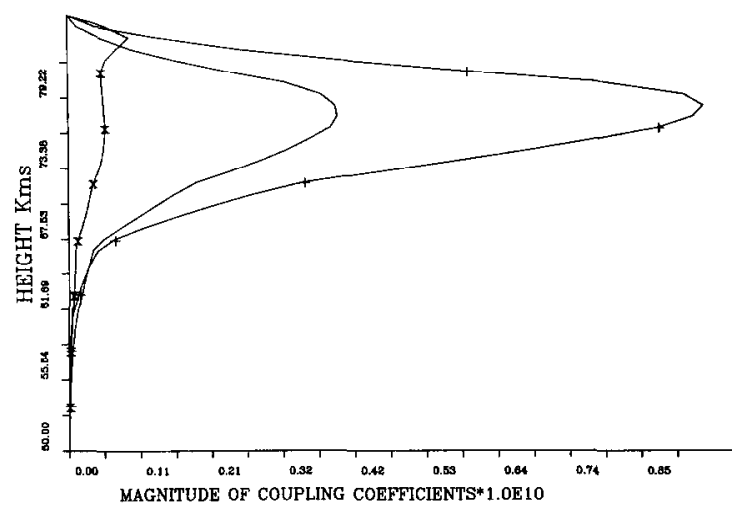

Fig. 6. Path NPM Hawaii-Palmer. Plots of the magnitudes of the diagonal elements of the scattering matrix $\eta^{\mathrm{mal}}$, evaluated at the centre of the LIE as functions of $z$. Results are presented for the three dominant TM modes $m=3$ (no mark), $m=2(x)$, and $m=5(+)$. Maximal scattering occurs in the height range $70-80 \mathrm{~km}$

called 'whispering gallery mode', peaks at over 80 $\mathrm{km}$, while the largest degree of 'diagonal' scattering is obtained for mode $m=5$. Figure 7 plots the phases of $\eta^{m m}$ as functions of $z$ for the three dominant TM modes $m=3,2,5$. As expected, at low altitudes $\sim 60$ $\mathrm{km}$ the phase is seen to be close to 225 degrees, except for the 'whispering gallery' mode $m=2$, for which the height gain function has a significant phase lag at this height. All the phases decrease with altitude, due to the phase lag contribution from the height gain function squared. In the altitude range from $70-80 \mathrm{~km}$, where scattering is largest, the scatter phases are in the range

NPM HAWAII-PALMER $23.4 \mathrm{kHz}$

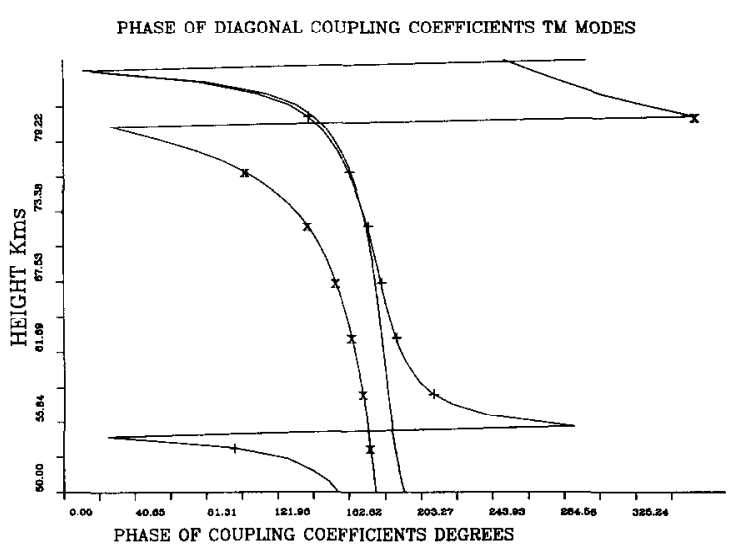

Fig. 7. Path NPM Hawaii-Palmer. Plots of the phases of the diagonal elements of the scatter matrix $\eta^{\mathrm{mm}}$, for dominant TM modes $m=3,2,5$, plotted at the centre of the LIE as functions of height $z$.
NPM HAWAII-PALMER $23.4 \mathrm{kHz}$ MAGNITUDE OF CROSS COUPLING COEFS - 3 TM MODES

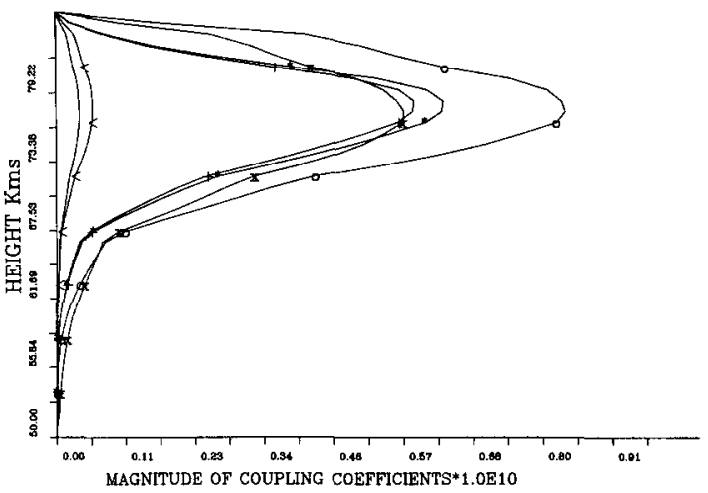

Fig. 8. Path NPM Hawaii-Palmer. Plots of the magnitudes of off-diagonal elements of the seatter matrix $\eta^{\text {mm' }}$, as functions of $z$. Elements plotted are 32(no mark), 23(X), 35( +), $53(*), 25(\mathrm{O})$, and $52(<)$. The magnitudes are comparable with those of the diagonal elements, suggesting that inter modal scattering is very important.

120-160 degrees, which is in rough agreement with the scatter phase of the Poulsen et al. (1993a,b) model. Figure 8 plots the magnitudes of 6 off-diagonal conponents of the scatter matrix $\eta^{m m^{\prime}}$. These are evaluated in the centre of the LIE and presented as functions of $z$. The elements shown correspond to scattering between dominant TM modes i.e. elements 32,23 , $35,53,25,52$ where, for example, $\eta^{32}$ indicates the magnitude of the scattering from TM mode $m=3$ to TM mode $m=2$. The magnitudes are clearly comparable with those of the diagonal elements, and maximum scattering is associated with the altitude range $70-80 \mathrm{~km}$ in this case. The highest values seem to be associated with scattering into a dominant mode $m=3$ from the other two, and with scattering from the dominant mode into the higher order mode $m=5$. It is apparent that intermodal scattering is very important. Since the LIE may actually be quite close to either the transmitter or the receiver, scattered higher order modes will significantly affect the resulting Trimpi. On the subject of intermodal scattering it is worth evaluating the $\bar{\eta}^{\left(\mathrm{mm}^{\prime}\right)}$ matrix at the LIE centre for the 3 dominant TM modes, which requires integration of $\eta$ over $z$. The result is, in arbitrary units

$$
\begin{gathered}
\bar{\eta}^{33}=-23.6-j 2.9, \quad \bar{\eta}^{32}=5.2-j 2.1, \\
\bar{\eta}^{35}=33.0+j 1.26 \\
\bar{\eta}^{23}=35-j 23.0, \quad \bar{\eta}^{22}=-1.8+j 9.4, \\
\bar{\eta}^{25}=-45.0+39 j \\
\bar{\eta}^{53}=36+j 4.4, \quad \bar{\eta}^{52}=-7.9+j 3.7, \\
\bar{\eta}^{55}=-52-j 2 .
\end{gathered}
$$




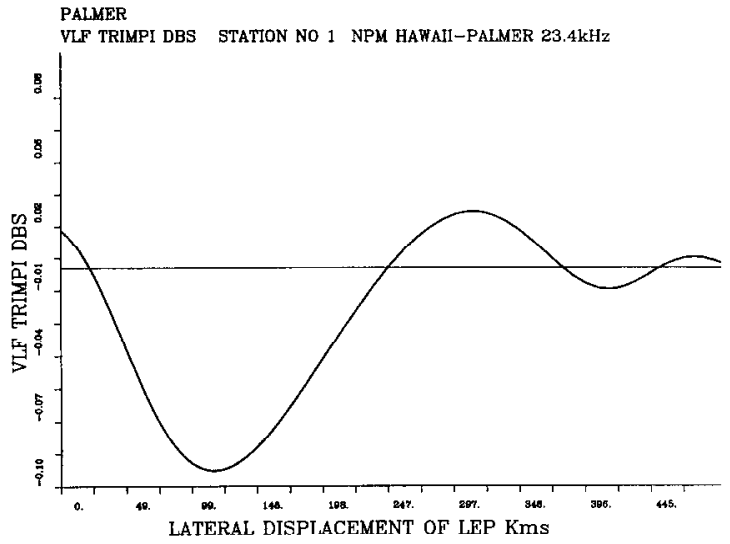

Fig. 9. Path NPM Hawaii-Palmer. Plot of amplitude Trimpi in $\mathrm{dB}$ as a function of lateral displacement of the LIE from the GC TX-RX path. Note the predominance of the classic $(-+)$ Trimpi at almost all displacements. The LIE is 9600 $\mathrm{km}$ from the transmitter and $2800 \mathrm{~km}$ from the receiver.

This matrix forecasts significant scattering between all three dominant TM modes, the only element which is small is that for scattering from the dominant mode $m=3$ into the whispering gallery mode $m=2$. Not only are the elements of the scattering matrix all large, they are also fairly sensitive to the choice of $\Delta N_{e}(z)$. They are thus erratic and hard to predict.

The LEP is somewhat arbitrarily placed on the GLC path a distance of $9600 \mathrm{~km}$ from NPM or 2800 $\mathrm{km}$ from Palmer station. Following Poulsen et al. $(1990,1993 a, b)$ the LEP is moved laterally from the GLC path a variable distance $d_{0}$. Figures 9 and 10 plot the amplitude and phase 'Trimpis' at Palmer as functions of displacement $d_{0}$. It is seen that the basic Trimpi type is the classic one of negative amplitude

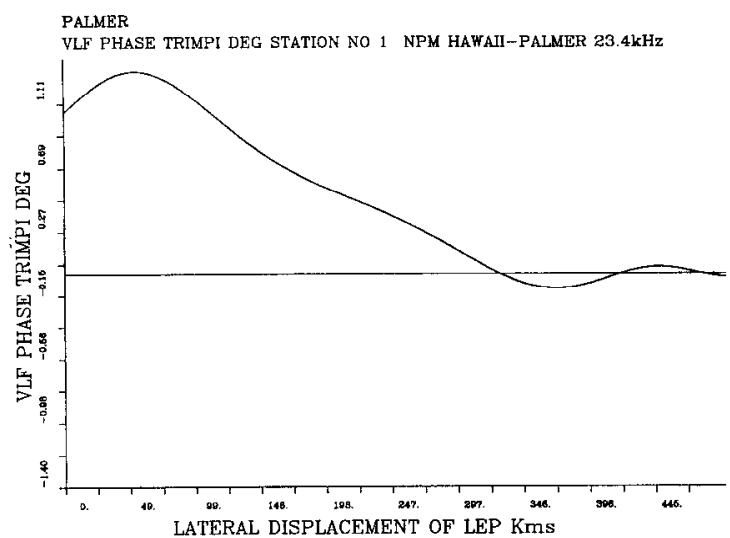

Fig. 10. Path NPM Hawaii-Palmer. Plot of phase Trimpi in degrees as a function of lateral displacement of the Trimpi from the TX-RX GLC path. perturbation and positive phase shift $(-+)$. This type occurs for almost all values of $d_{0}$, which is in good agreement with observations on this path which show that type $(-+)$ is dominant.

With increasing $d_{0}$ the Trimpi amplitudes fall quickly. This is expected and due to the fact that the radiation pattern of the scattered field is strongly focussed in the direction TX-LIE. With increasing $d_{0}$ the scatter phase winds round in a negative direction due to the travel delay. Trimpis of type $(++)$ and then $(+-)$ occur at displacement ranges $\sim 250-300$ $\mathrm{km}$, but the corresponding Trimpi magnitudes are so small that they would probably be unobservable. Close inspection of the curves shows some evidence of multimodal effects and intermodal scattering. Certainly the LIE will radiate significant energy in the three dominant TM modes, and these modes will all be significant at Palmer which is only $2800 \mathrm{~km}$ from the LIE.

One of the key postulates of the University of Otago group was that off GC path scattering was significant. The LIE modelled here is quite large and has no fine structure. Its horizontal shape, however, has quite sharp edges. Figure 11 plots the radiation pattern of the LIE, where the distance from NPM to the LIE is taken to be $6000 \mathrm{~km}$ and the distance from the LIE to the observation point is $6000 \mathrm{~km}$. The heavy curve shows the total radiated amplitude in $\mathrm{mV} / \mathrm{m}$ as a function of scattering angle. The half beamwidth of the scattered field is about 5 degrees. The other three curves show the separate radiation patterns of each of the three dominant TM modes. Not surprisingly, that for mode $m=3$ is the largest, with modes $m=2,5$

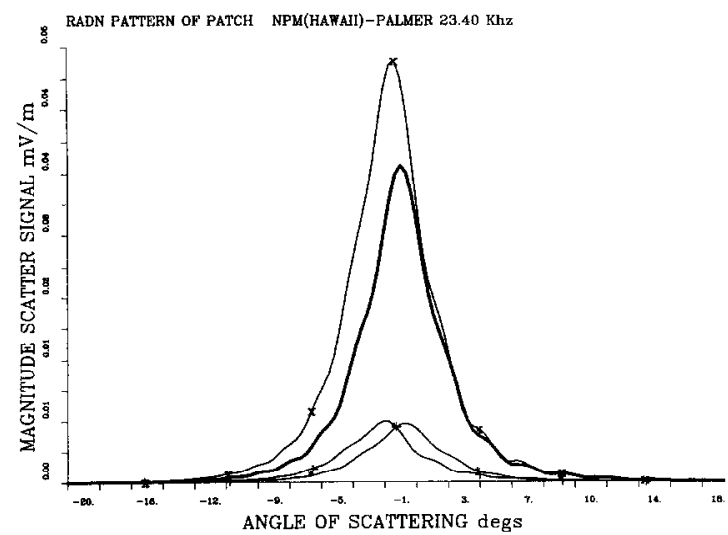

Fig. 11. Path NPM Hawaii-Palmer. Radiation pattern of the LIE, in electric field magnitude $\mathrm{mV} / \mathrm{m}$, against scattering angle. The ranges TX-LIE and LIE-RX are both $6000 \mathrm{~km}$. The heavy curve is the total radiated field (modal sum). The light curves are radiation patterns of individual dominant TM modes, $m=3(\mathrm{X}), m=2(+)$ and $m=5\left(^{*}\right)$. 
NWC to Dunedin great circle path

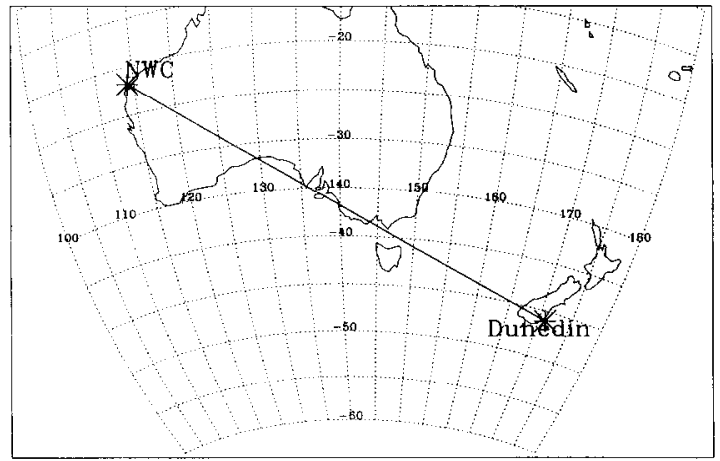

Fig. 12. Map of path NWC (Australia)-Dunedin N.Z.

about $1 / 6$ as large. Smaller LIE's or LIE's with fine structure would have a much wider scattering widths.

\section{Path NWC(Australia)-Dunedin N.Z.}

The second path to be modelled is from the high power VLF facility NWC on the NW coast of Australia to Dunedin in New Zealand. The geographic map of this path is shown in Fig. 12. The path length is $5745 \mathrm{~km}$ and is partly over land and partly over water. NWC transmits 2 close frequencies, at 22250 and $22350 \mathrm{~Hz}$, and the Trimpis will be calculated for both of these frequencies. MODEFNDR returns 12 allowable modes for this scenario, but again the dominant modes are TM modes $m=3, m=2$ (the whispering gallery mode) and $m=5$. Ground parameters are assumed to be those for sea water. Ionospheric parameters $\beta=0.46$ and $h^{\prime}=88 \mathrm{~km}$ are selected. The LIE is again assumed to be elliptical, orientated magnetic $\mathrm{E}-\mathrm{W}$, and with a major axis $a=250 \mathrm{~km}$ and minor axis $50 \mathrm{~km}$. The assumed vertical profiles of the unperturbed electron density $N_{e}(z)$ and total perturbed electron density, $\Delta N_{e}(z)+N_{e}(z)$, are shown in Fig. 13. This profile is plausible but inevitably somewhat arbitrary. The profile of $\Delta N_{e}(z)$ in the horizontal plane is again a Butterworth function of order 10 . Turning to the results, Fig. 14 shows the equivalent vertical conductivity $\sigma_{z=}$ at the LIE centre, plotted as a function of $z$. The magnitude peaks at about $80 \mathrm{~km}$. The phase is close to zero at low altitudes, where the LIE appears purely resistive. At $80 \mathrm{~km}$ there is a phase lag of about 5 degrees, which corresponds to a negative susceptibility and is what one expects from free electron motion in the presence of collisional damping.

Figure 15 plots the magnitudes of three diagonal components of the scattering matrix $\eta^{\mathrm{mm}}$ at the centre of the LIE. The curves are presented as functions of $z$ for the three dominant TM modes. They are very

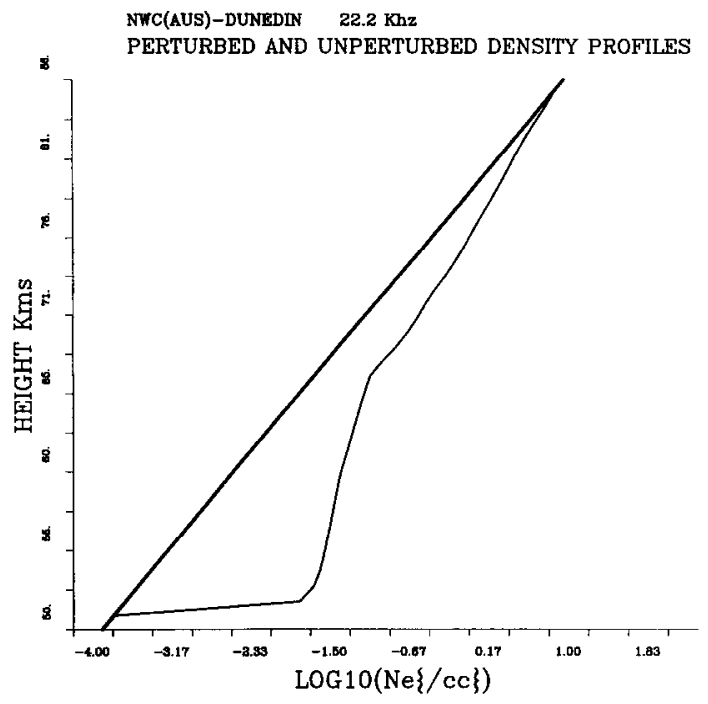

Fig. 13. Path NWC-Dunedin. Plot of unperturbed density $N_{e}(z)$ and total perturbed density $N_{e}(z)+\Delta N_{e}(z)$, expressed as $\log$ to base 10 of density in electrons $\mathrm{cm}^{-3}$.

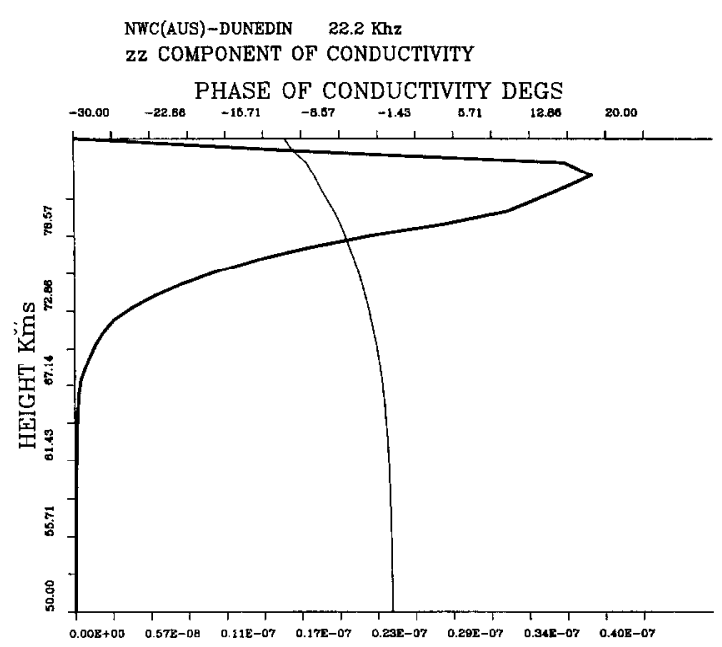

CONDUCTIVITY MAGNITUDE Mho/m

Fig. 14. Path NWC-Dunedin. Plot of equivalent vertical conductivity $\tilde{\sigma}_{33}(z)$ as a function of $z$, measured at the centre of the LIE. The magnitude (heavy curve) peaks at about 80 $\mathrm{km}$. The phase is close to zero, showing resistive behaviour, but increasing phase lag of order 5 degrees will be noted at high altitudes.

similar to those for NPM Hawaii-Palmer. Most diagonal scattering occurs in the height range $70-80 \mathrm{~km}$. Figure 16 shows the phases of these diagonal components as a function of $z$. The phases are close to 225 degrees at low altitude, as expected theoretically, and decrease steadily with altitude. Figure 17 plots magnitudes of the six most important off-diagonal com- 
NWC(AUS)-DUNEDIN $22.3 \mathrm{kHz}$ DIAGONAL COUPLING COEFFICIENTS 3 DOMINANT TM MODES

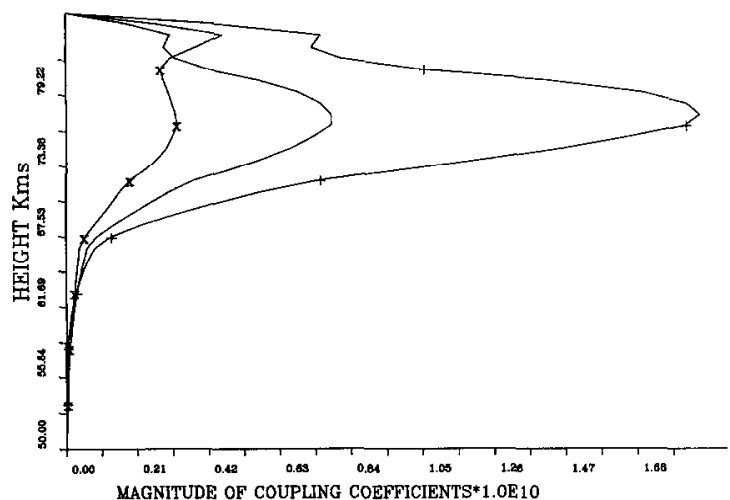

Fig. 15. Path NWC-Dunedin. Magnitudes of diagonal components of the scattering matrix $\eta^{\mathrm{mm}}$ for the three dominant TM modes $m=3$ (no mark), $m=2(X)$ and $m=5(+)$. Maximum scattering occurs in the height range $70-80 \mathrm{~km}$.

ponents of the scattering matrix $\eta^{m m}$, which are $\eta^{32}$, $\eta^{23}, \eta^{35}, \eta^{53}, \eta^{25}, \eta^{52}$. Once again the off diagonal elements are of the same order as the diagonal terms, pointing to strong inter-modal scattering among the dominant TM modes. These terms peak at about 75 $\mathrm{km}$ altitude. To calculate the Trimpis at Dunedin the LEP is placed on the GC path at $1600 \mathrm{~km}$ from NWC. Figures 18 and 19 plot the magnitude and phase Trimpis at Dunedin as functions of $d_{0}$, the lateral displacement of the LIE from the GC path. Trimpis are shown for both frequencies transmitted by NWC,

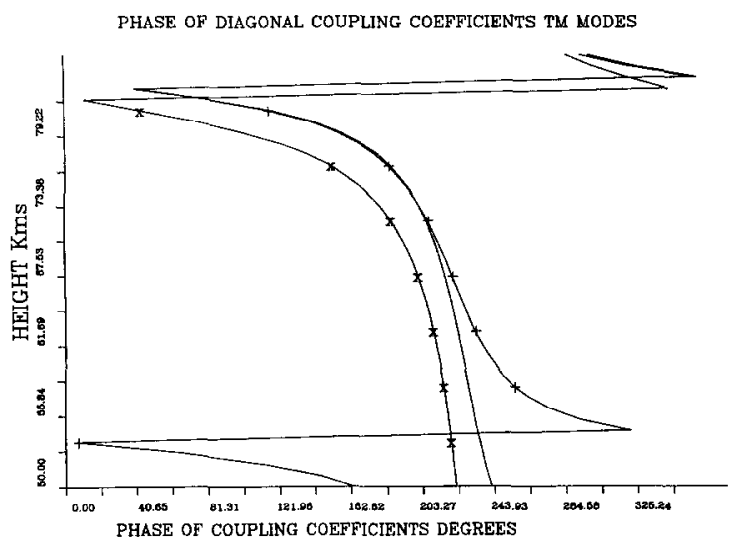

Fig. 16. Path NWC-Dunedin. Plots of the phases of the diagonal elements of the scatter matrix $\eta^{\mathrm{mm}}$, for dominant TM modes $m=3,2,5$. The data is plotted for the centre of the LIE as functions of height $z$. The basic scatter phase is $\sim 225$ degrees at low altitudes where the LIE responds as a resistance. The phase decreases with altitude, due to phase lags in the height gain function squared.
NWC(AUS)-DUNEDIN $22.3 \mathrm{kHz}$

MAGNITUDE OF CROSS COUPLING COEFS - 3 TM MODES

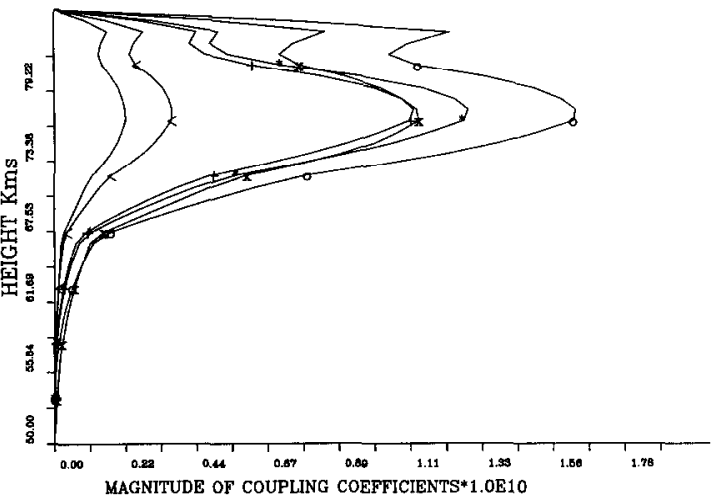

Fig. 17. Path NWC-Dunedin. Plots of magnitudes of offdiagonal elentents of the scatter matrix $\eta^{\mathrm{mm}^{\prime}}$, as functions of z. Elements plotted are 32(no mark), 23(X), 35(+), 53(*), $25(\mathrm{O})$, and $52(<)$. The magnitudes are comparable with those of the diagonal elements, suggesting that inter-modal scattering is very important.

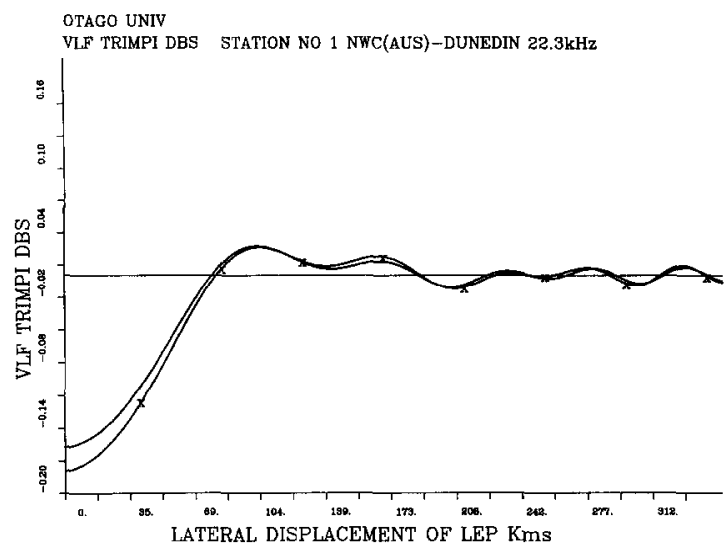

Fig. 18. Path NWC-Dunedin. Plot of amplitude Trimpi at Dunedin as a function of $d_{0}$, the lateral displacement of the LIE from the GLC path TX-RX. The classic $(-+)$ Trimpi is predominant. Other Trimpi types at large displacements would not be observable. Results are plotted for both frequencies transmitted by NWC, $f=22250 \mathrm{~Hz}$ (no mark) and $f=22350 \mathrm{~Hz}(\mathrm{X})$.

though these are very similar. The dominant Trimpi type is again the classic $(-+)$, with positive phase and negative amplitude perturbations. Only at large displacements, $d_{0}$, do other types appear, although these have small amplitudes and would probably not be observed. For the case $d_{0}=0$ (GC path) the scatter phase of the dominant mode is in the region of 160 degrees, somewhat different from the Poulsen et al. $(1993 \mathrm{a}, \mathrm{b})$ phase of 135 degrees. The result is a relatively large magnitude Trimpi and a small phase 


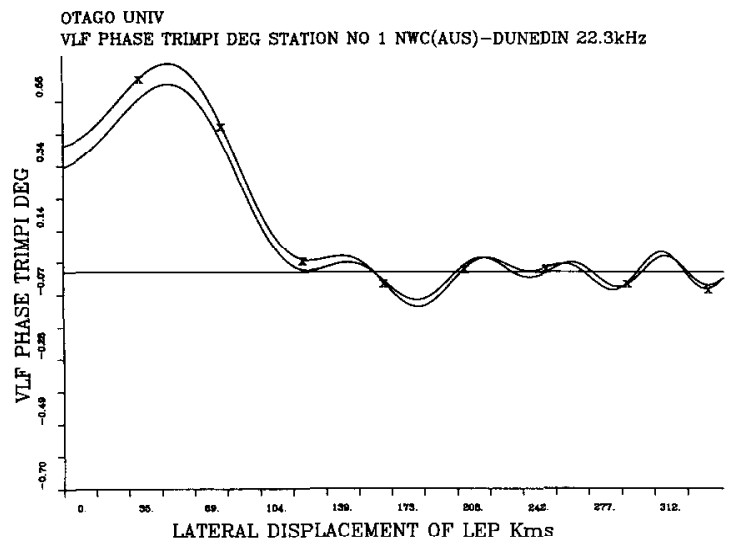

Fig. 19. Path NWC-Dunedin. Plot of phase Trimpi at Dunedin, shown as a function of lateral displacement $d_{0}$.

Trimpi. Examination of the curves reveals evidence of multimodal effects as well as of intermodal scattering. This conclusion is confirmed by inspection of the radiation patterns of the LIE, shown in Fig. 20, which are calculated assuming a TX-LIE distance of $3000 \mathrm{~km}$ and a LIE-RX distance of $3000 \mathrm{~km}$. This figure presents the radiation pattern of the scattered field in $\mathrm{mV} / \mathrm{m}$, for the total field and for each of the dominant TM modes separately. The half beamwidth is again about 5 degrees. The dominant mode $m=3$ has the highest amplitude in the scattered field, but $m=2$ and $m=5$ have amplitudes each about $1 / 3$ of that of $m=3$. This surely confirms the multimodal character of this scenario. Also, since the NWC-LIE distance is

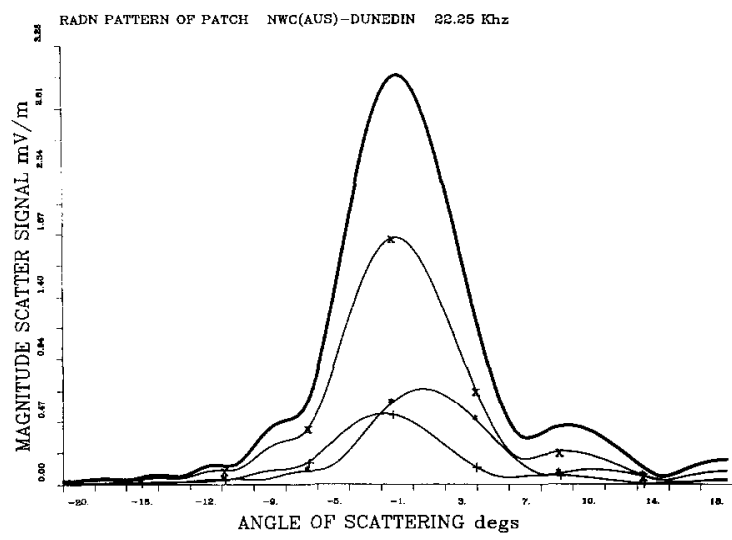

Fig. 20. Path NWC-Dunedin. Magnitude of scattered VLF radiation in $\mathrm{mV} / \mathrm{m}$, as a function of scattering angle. The ranges TX-LIE and LIE-RX are both assumed to be 2800 $\mathrm{km}$. The heavy curve is the total field (modal sum). The light curves are the radiation patterns for each TM mode, $m=3(\mathrm{X}), m 2(+)$ and $m=5\left(^{*}\right)$. The scattered energy in modes $m=2,5$ is seen to be significant. small, the incident field $E_{0}$ at the LIE will be highly multimodal, and the cross scattering into the dominant TM modes will be significant.

In experimental reality the path NWC-Dunedin is complicated by the effect of the Southern Alps on the propagation paths, as well as the mixed land/sea nature of all the long paths. More detailed comparison with the data for this path requires a version of the code using LWPC.

\section{Path Omega (Australia)-Dunedin N.Z.}

The transmitter Omega Australia is located on the South East coast of Australia. The transmission path to Dunedin N.Z. is $2096 \mathrm{~km}$ and is mainly over the Tasman Sea, but the last $150 \mathrm{~km}$ are over the alpine range of the Southern Alps. This short path length makes the 'Trimpi' problem very complex and dominated by several modes, all of comparable magnitudes. Omega (Australia) transmits intermittently on 5 well separated frequencies, which are 13600,13000 , 11333,10500 and $10200 \mathrm{~Hz}$. This makes the path Omega(Aus) Dunedin a useful one for LIE imaging and for diagnostic purposes. The frequencies are somewhat lower than those of NPM/NWC and thus the scattering problem becomes rather different. Ground parameters selected are those for seawater, and ionospheric parameters are chosen to be $h^{\prime}=86$ $\mathrm{km}, \beta=0.48$. The geographical map of the path is shown in Fig. 21.

At these lower frequencies MODEFNDR returns six allowable modes of which $m=1,3,5$ are the dominant TM modes. The LIE is assumed to be elliptical in shape and orientated magnetic E-W. The major axis $a=300 \mathrm{~km}$ and the minor axis $b=100 \mathrm{~km}$. The selected unperturbed electron density profile $N_{e}(z)$ and the perturbed total profile $N_{e}(z)+\Delta N_{e}(z)$ are shown in

\section{Omega Australia to Dunedin}

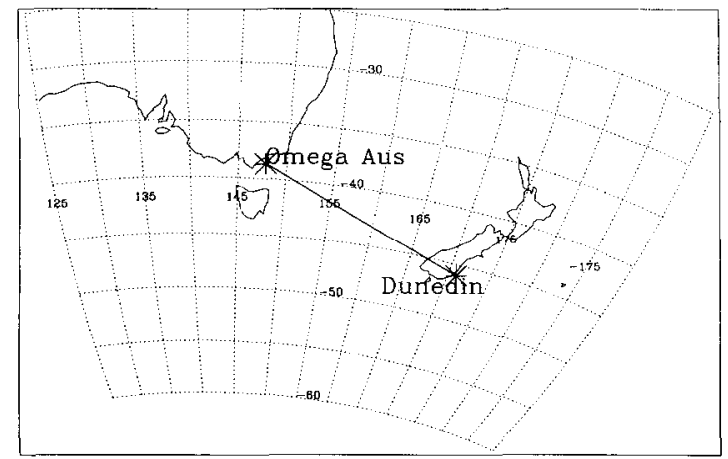

Fig. 21. Map of path Omega(Australia)-Dunedin, N.Z. 


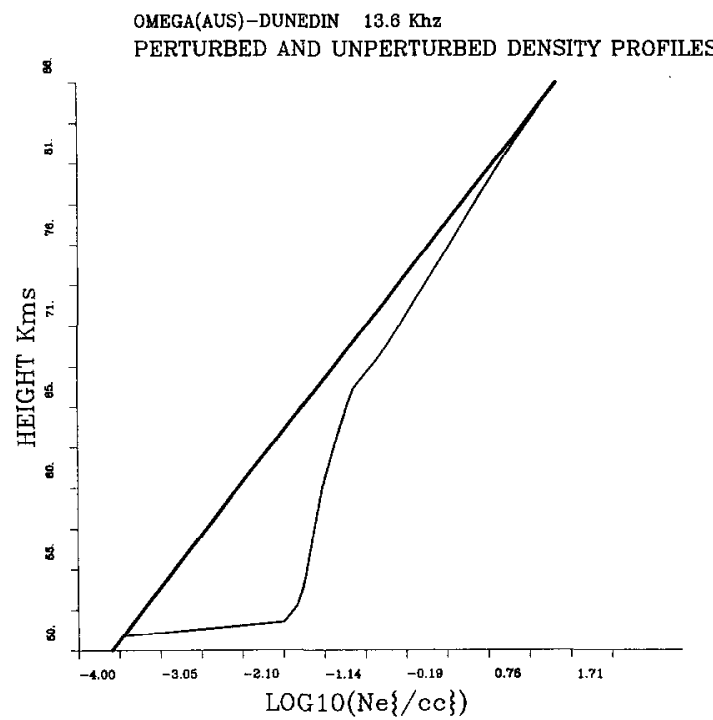

Fig. 22. Path Omega(Australia)-Dunedin. Plot of unperturbed electron density profile $N_{l}(z)$ and total electron density $\left\{N_{e}(z)+\Delta N_{t}(z)\right\}$, expressed as $\log$ to base 10 of density in electrons $\mathrm{cm}^{-3}$.

Fig. 22. The horizontal variation of $\Delta N_{e}$ across the LIE ellipse is described by a Butterworth function of order 10.

The $z$ dependence of equivalent vertical conductivity $\tilde{\sigma}_{33}(z)$ at the LIE centre, calculated at $f=13600 \mathrm{IIz}$, is shown in Fig. 23. A pronounced maximum occurs at $80 \mathrm{~km}$. The conductivity phase is close to zero at low altitudes, but above $80 \mathrm{~km}$ a

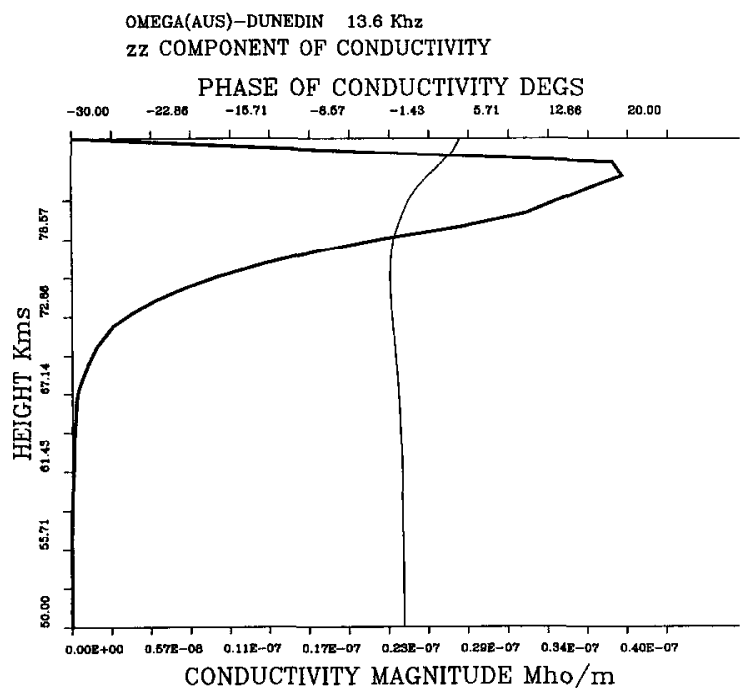

Fig. 23. Path Omega(Australia)-Dunedin. Plot of magnitude (heavy curve) and phase of equivalent vertical conductivity $\tilde{\sigma}_{33}(z)$, measured at the LIE centre, at $f=13600 \mathrm{~Hz}$. positive phase of a few degrees is noted, suggesting that at these low frequencies the plasma behaves like a lossy dielectric.

Figure 24 plots the magnitudes of three diagonal components of the scatter matrix $\eta^{\mathrm{mm}}$, evaluated at the LIE centre for the three dominant TM modes $m=1,3,5$. These curves are for $f=13600 \mathrm{~Hz}$ only, and plotted as functions of $z$. They show that the dominant scattering is in the altitude range $70-80 \mathrm{~km}$, and that the magnitude of the scattering from mode $m$ to mode $m$ increases sharply with mode order $m$. Figure 25 plots the phases of $\eta^{m m}$ for these modes. As expected at low altitudes all the phases are $\sim 225$ degrees and decrease monotonically with $z$. At $75 \mathrm{~km}$, the height of maximum scattering, the phases of all 3 dominant TM modes are of order 180 degrees for this particular scenario. Figure 26 plots the magnitudes of the six dominant off diagonal elements of $\eta^{m m}$, corresponding to scattering from mode $m$ to mode $m^{\prime}$. Concentrating again on scattering between TM modes $m=1,3,5$, the coefficients plotted are $\eta^{13}, \eta^{31}, \eta^{15}, \eta^{51}$, $\eta^{35}$ and $\eta^{53}$. These are seen from the graph to be all of the same order of magnitude as the diagonal elements of $\eta$, and thus again inter-modal scattering is very significant. The largest inter-modal scattering is from mode 5 to mode 3 , followed by scattering from mode 1 to 5 and mode 1 to 3 . It should be pointed out that the scatter matrix is a sensitive function of the $\Delta N_{c}(z)$ profile. It is of some interest to examine the modal coupling matrix $\bar{\eta}$ evaluated at the LIE centre at $f=13600 \mathrm{~Hz}$. The terms corresponding to the dominant TM modes $m=1,3,5$ are, in arbitrary units

$$
\begin{gathered}
\bar{\eta}^{11}=-8.5-j 1.47, \quad \bar{\eta}^{13}=21+j 2, \\
\bar{\eta}^{15}=-28-j 1.6 \\
\bar{\eta}^{31}=12+j 1.9, \quad \bar{\eta}^{33}=-31.6-j 2.6, \\
\bar{\eta}^{35}=42+j 1.4 \\
\bar{\eta}^{51}=-19-j 3.8, \quad \bar{\eta}^{53}=50+j 5.9, \\
\bar{\eta}^{55}=-68-j 5
\end{gathered}
$$

To calculate Trimpis observed at Dunedin, the LIE is placed, somewhat arbitrarily on the GC path a distance of $800 \mathrm{~km}$ from Omega(Australia) in the Tasman Sea, which point is $1296 \mathrm{~km}$ from Dunedin. The LIE is displaced laterally a distance of $d_{0} \mathrm{~km}$. Magnitude and phase Trimpis at Dunedin are plotted in Figs 27 and 28 as functions of $d_{0}$, for all five frequencies transmitted by Omega. The multimodal nature of the problem is very clear, and the classic $(-+)$ Trimpi is no longer dominant. All types of Trimpi occur, and interpretation of the results becomes very complex. At $13600 \mathrm{~Hz}$ the Trimpi is negative phase 
DIAGONAL COUPLING COEFFICIENTS 3 DOMINANT TM MODES

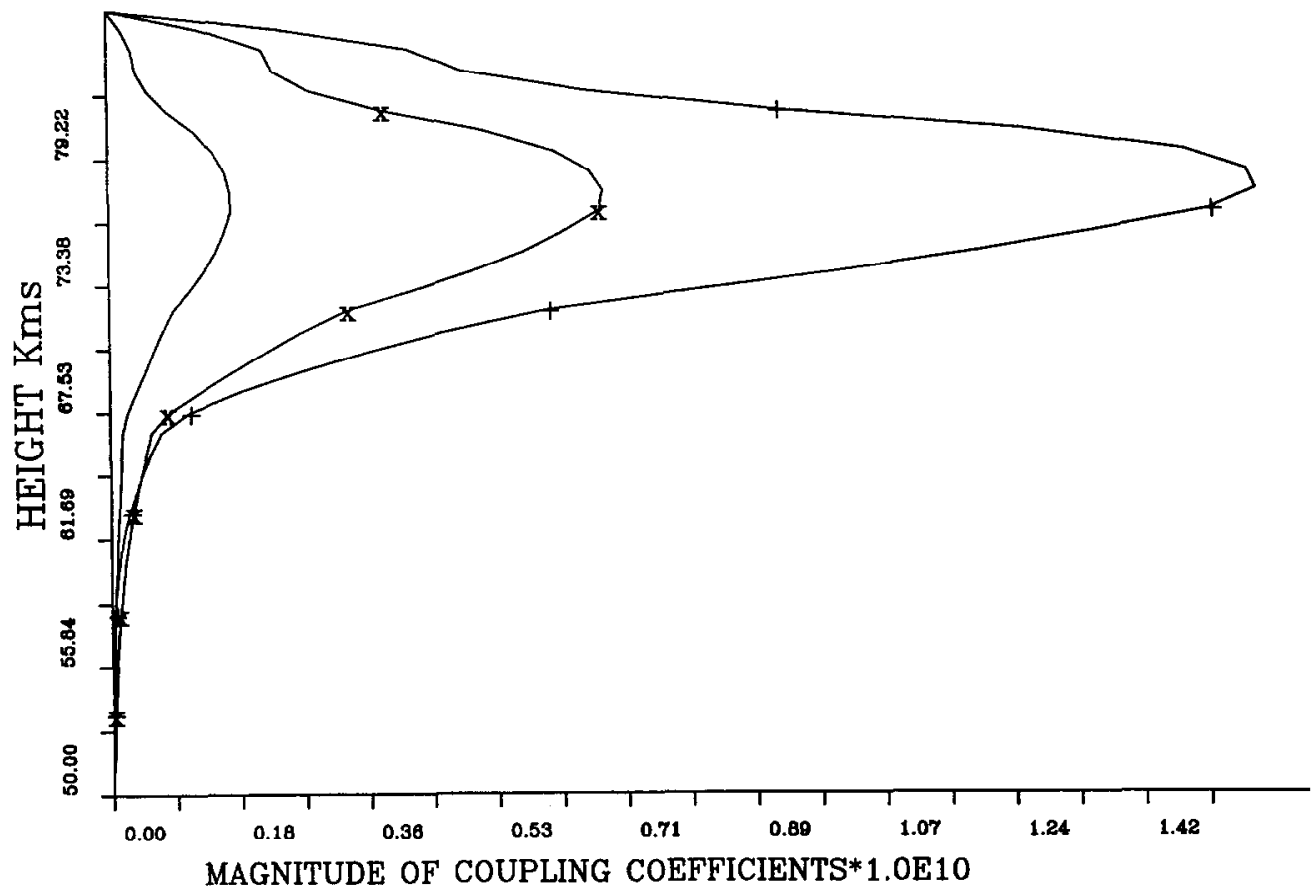

Fig. 24. Path Omega(Australia)-Dunedin. Magnitudes of diagonal elements of the scatter matrix $\eta^{\mathrm{mm}}$ for the three dominant TM modes $m=1$ (no mark), $m=3(\mathrm{X})$ and $m=5(+)$, calculated at $f=13600 \mathrm{~Hz}$. Maximum scattering occurs at $75 \mathrm{~km}$ altitude.

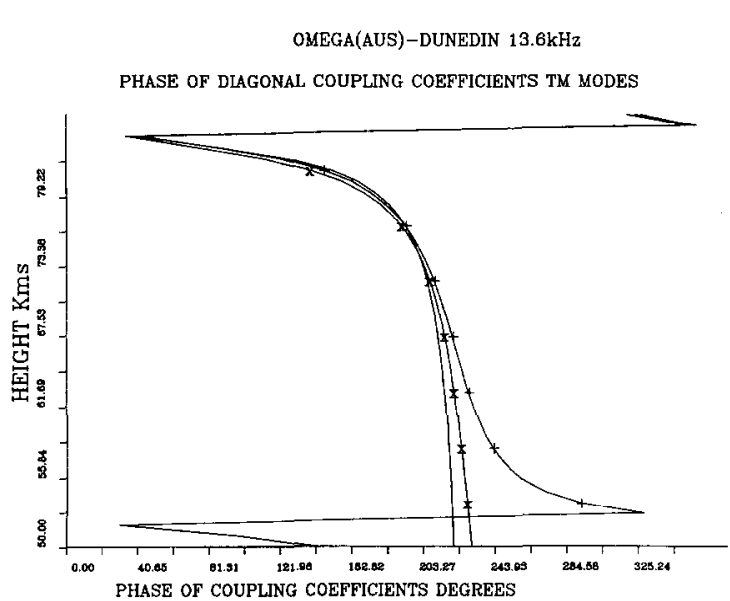

Fig. 25. Path Omega(Australia)-Dunedin. Plot of the phases of the diagonal elements of the scatter matrix $\eta^{\mathrm{mm}}$, for the three dominant TM modes, at $f=13600 \mathrm{~Hz}$. At low altitudes the phase is $\sim 225$ degrees, and decreases monotonically with altitude.
OMEGA(AUS)-DUNEDIN $13.6 \mathrm{kHz}$ MAGNITUDE OF CROSS COUPLING COEFS - 3 TM MODES

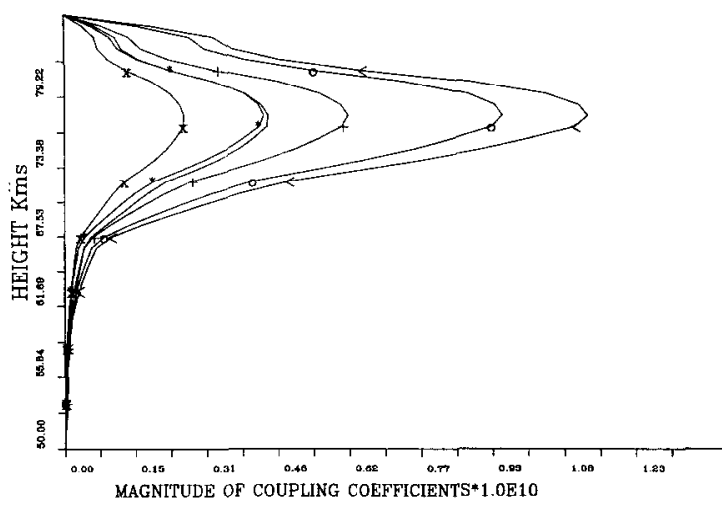

Fig. 26. Path Omega(Australia)-Dunedin. Plots of the magnitudes of off-diagonal elements of the scatter matrix $\eta^{\mathrm{mm}}$, as functions of $z$, calculated at $f=13600 \mathrm{~Hz}$. Elements plotted correspond to the dominant TM modes and are 13(no mark), $31(\mathrm{X}), 15(+), 51\left(^{*}\right), 35(\mathrm{O})$ and $53(<)$. The magnitudes of the off-diagonal elements are of the same order as the diagonal elements and thus inter-modal scattering is very significant. 


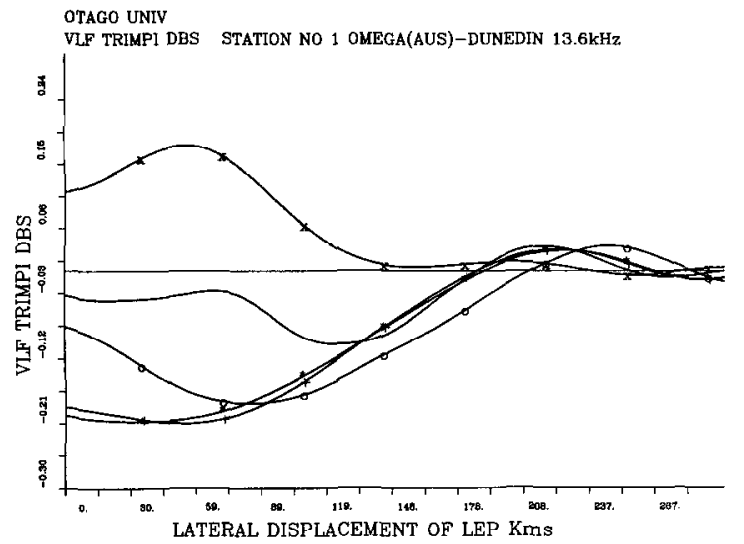

Fig. 27. Path Omega(Australia)-Dunedin. Plot of amplitude Trimpis at Dunedin, plotted as functions of $d_{0}$, the lateral displacement of the LIE from the GC path. Multimodal effects are very pronounced, and all scatter phases are observed. The curves shown are for the 5 frequencies transmitted by Omega i.e. $13600 \mathrm{~Hz}$ (no mark), $13000 \mathrm{~Hz}(\mathrm{X})$, $11333 \mathrm{~Hz}(+), 11050 \mathrm{~Hz}\left(^{*}\right)$, and $10200 \mathrm{~Hz}(\mathrm{O})$.

for $d_{0}<100 \mathrm{~km}$, but the type becomes $(-+)$ for $d_{0}>100 \mathrm{~km}$. At $13000 \mathrm{~Hz}$ the observable Trimpi type is positive amplitude negative phase $(+-)$, regardless of $d_{0}$. At this frequency the Trimpi magnitudes are quite large, because Dunedin is close to a modal interference minimum for the direct signal, which makes the direct signal small and the effect of any scattered signal relatively large. At $11333 \mathrm{~Hz}$ the Trimpi type is the classic $(-+)$ for all observable ranges, and the same is true for $11050 \mathrm{~Hz}$ and $10200 \mathrm{~Hz}$.

It is quite interesting to plot the radiation pattern of the LIE for a particular frequency, here chosen to be $13600 \mathrm{~Hz}$. We assume a TX-LIE distance of 1000

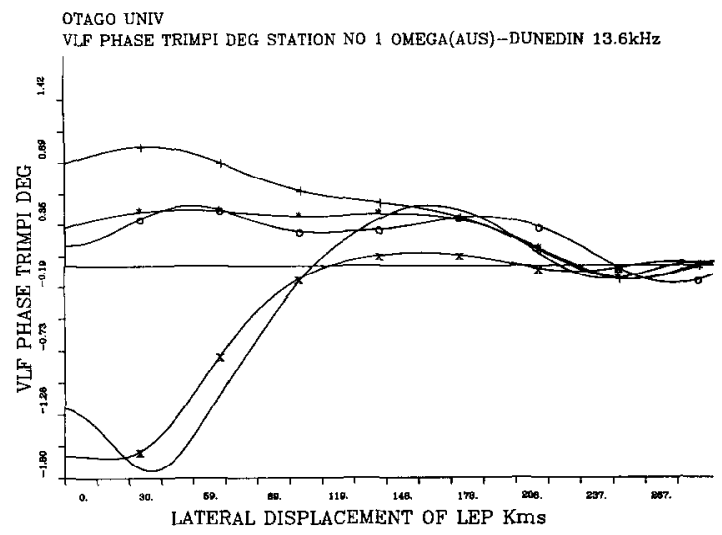

Fig. 28. Path Omega(Australia)-Dunedin. Phase Trimpis at Dunedin, plotted as functions of $d_{0}$, for all five frequencies transmitted by Omega.

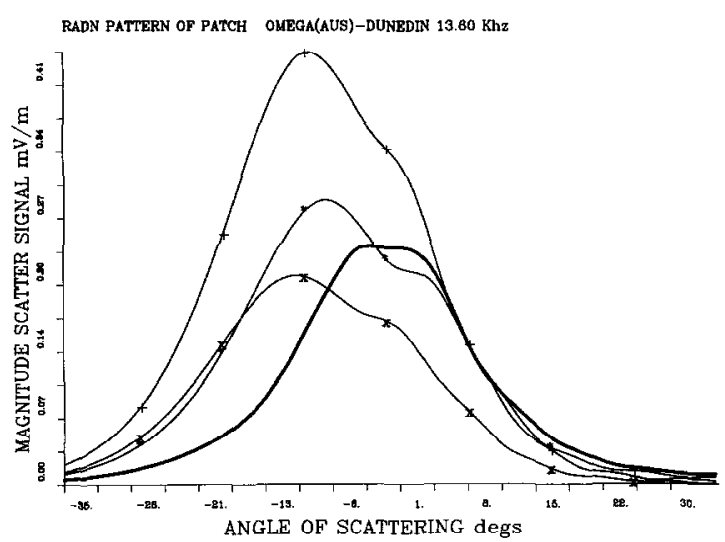

Fig. 29. Path Omega(Australia)-Dunedin. Magnitude of scattered radiation from the LIE as a function of scattering angle, in $\mathrm{mV} / \mathrm{m}$, evaluated at $f=13600 \mathrm{~Hz}$. The ranges TX LIE and LIE-RX are both assumed to be $1000 \mathrm{~km}$. The heavy curve is the total field (modal sum). The light curves are for each dominant TM mode separately $m=1(\mathrm{X}), m=3(+)$ and $m=5\left(^{*}\right)$. Note the skewed distribution for modal radiation patterns and also evidence of intermodal cancellation in the scattered radiation.

$\mathrm{km}$ and a LIE-RX distance of $1000 \mathrm{~km}$. Figure 29 plots the scattered field amplitude in $\mathrm{mV} / \mathrm{m}$ as a function of scattering angle. The heavy curve is the total scattered field (modal sum) and shows a half width of about 12 degrees, obviously greater than for NPM and NWC because of the larger wavelength. For the same LIE Omega signals will give more off axis scattering and will thus perform better for localisation purposes. The three light curves are the radiation patterns for each of the dominant TM modes separately. Clearly the phasing of the scattered modal signals is such that intermodal cancellation effects are significant. The scattered mode $m=3$ seems to have the largest amplitude, but there is significant power in all three modes. Interestingly the radiation patterns of the individual modes are skewed away from the TXLIE direction. The radiation pattern will not be symmetrical about the TX-LIE direction for short ranges and large LIE's.

We conclude with one general comment on these results. The overall magnitude of Trimpi cvents obtained in these calculations is realistic, typically 1 degree and/or about $0.2 \mathrm{~dB}$. Some observed Trimpis are somewhat larger than this. These must arise either from patches that are close to the receiver or transmitter, from large patches, or from patches with larger electron density perturbations. A considerable amount of controversy has been generated over the question of how important off-GC path scattering is. For the Trimpis modelled here the scattering width of 
the patches is always less than 5 degrees, except for Omega Australia where the figure is nearer 15 degrees. The University of Otago group (Dowden and Adams, 1990, 1993a) report observational evidence of wide scattering angles and very large Trimpis, in a number of cases. Such observations of wide scattering angles must be due to

(a) very 'strong' patches, rendering widely scattered signals more visible,

(b) scattering from one or more small intense patches, or

(c) fine horizontal structure in the function $\mathrm{d} N_{e}(x$, $y, z)$.

Further research into this area must be the subject of future papers.

\section{CONCLUSIONS}

The Trimpi effect manifests itself as a small perturbation in received amplitude and phase of a sub ionospheric VLF transmission. These perturbations are due to scattering of VLF radiation from localised $D$ region inhomogeneities resulting mainly from precipitation of energetic electrons by lightning whistlers. In this paper we have developed a theoretical analysis and modelling formalism based upon a point scattering approach. The LIE is viewed as an assembly of point scatterers. The field $E_{0}$ incident upon each element of the LIE is calculated using VLF mode theory, and using the equivalent local conductivity matrix $\tilde{\sigma}$, the equivalent source current field $I_{\text {cff }}$ is calculated. Again modal propagation theory is used to calculate the scattered VLF field, which is sourced upon $J_{\text {eff }}(r)$. The modelling code naturally uses all allowable VLF modes throughout. The formalism does not require that the dimensions of the LIE be $\gg \lambda$, and it predicts strong inter-modal scattering at the LIE.

A numerical solution of the appropriate equations is virtually impossible unless the Born or weak scattering approximation is made. The Born approximation is valid for most observed Trimpi events and is applied by assuming that the field incident on each element of the LIE is that appropriate to the homogeneous problem. The electric polarisation field induced in the LIE can, to some extent, be accommodated by requiring that $D^{\prime}=0$ in the LIE rather than $E^{\prime}=0$.

The results of the modelling for the three chosen paths qualitatively agree reasonably well with values derived from observations, considering the lack of knowledge of LIE profiles, size, location and structure. The localised inhomogeneity may have perturbations in both electron density and collision frequency or temperature. The code uses all allowable modes throughout and is thus ideally suited also to modelling of short range Trimpi phenomena such as the Barr et al. $(1984,1985)$ heater experiment. The whole of the numerical and theoretical structure gives insight into the scattering process and reveals explicitly the dependence of scatter phase upon the $\Delta N_{e}(z)$ profile. Since $\Delta N_{e}(z)$ will be a function of time as the LIE decays, due to collisional recombination or cooling, a characteristic time profile of a Trimpi may be calculated for comparison with data.

The theory and numerical calculations predict substantial inter-modal scattering between dominant TM modes. The magnitude of the coupling depends sensitively on the profile $\Delta N_{e}(z)$ and is difficult to predict. There is little doubt that inter-modal scattering will be significant in most Trimpi scenarios, since the patch must be at short to medium range from either the transmitter or the receiver.

Much further work remains to be done on the Trimpi problem, mainly in the way of tightening up the model and making the calculations more rigorous. Work is currently underway at Southampton University by $\mathrm{R}$ Yeo; this includes

(a) use of the whole $\tilde{\sigma}$ matrix rather than just $\tilde{\sigma}_{33}$,

(b) use of the LWPC global VLF propagation package for all long transmission paths,

(c) simulation of multiple Trimpi events and the generation of scatter plots of the kind used by Dowden for data presentation,

(d) use of a time dependent profile $\Delta N_{e}(z)$ profile to generate characteristic time signatures of Trimpis.

Other possibilities for future developments include

(a) use of accurately calculated $\Delta N_{e}(z)$ profiles derived from VLF particle precipitation codes,

(b) simulation of the heater experiments,

(c) insertion of realistic horizontal fine structure into the LIE.

Perhaps the most urgent software update required is to modify or rewrite LWPC to give height gain functions that are accurate at high altitudes $>85 \mathrm{~km}$ and thus allow computation of the scattering from these altitudes.

Although the Born approximation is generally good for most Trimpi events, it begins to be inaccurate for large patches, or indeed for very strong ones. In the case of large patches two obvious remedies are available. We can assume that the phase of the $m$ th modal component of incident field $E_{0}$ is the WKB phase calculated in the inhomogeneous environment. We could assume that the vertical profiles of each incident mode $m$ are the height gain functions computed for 
the local perturbed ionospheric profile. Implementation of these refinements would be straightforward in principle, but tedious to program and expensive in terms of runtime required.

Acknowledgements-The author wishes to thank Prof. J. A.
Ferguson at NOSC, San Diego, for permission to use MODEFNDR software. Thanks are also owed to Professor R.L. Dowden for his help and advice during a visit to the University of Otago on a Beverley fellowship. The author also thanks Mr Richard Yeo for producing the data and geographic maps. British Antarctic Survey at Cambridge are acknowledged for the use of their VLF data base from Faraday, Antarctica.
Adams C. D. D. and Dowden R. L.

Baba K. and Hayakawa M.

Barr R., Rietveld M. T., Kopka H. and Stubbe P.

Barr R., Rietveld M. T., Stubbe P. and Kopka H.

Budden K. G.

Carpenter D. L. and LaBelle J. W.

Carpenter D. L., Inan U. S. and Smith A. J.

Carpenter D. L., Inan U. S., Paschal E. W and Smith A. J.

Carpenter D. L. and Inan U. S.

CCIR XVIIth Plenary Assembly, Dusseldorf

Clark T. D. G. and Smith A. J.

Cotton P. D. and Smith A. J.

Dowden R. L. and Adams C. D. D.

Dowden R. L. and Adams C. D. D.

Dowden R. L. and Adams C. D. D.

Dowden R. L. and Adams C. D. D.

Dowden R. L., Adams C. D. D., Rietveld M. T., Stubbe P. and Kopka H.

\section{REFERENCES}

1990 VLF group delay of lightning induced electron precipitation echoes from measurement of phase and amplitude perturbations at two frequencies. J. Geophysical Research 95, 2457-2462.

1995 The effect of local ionospheric perturbations on subionospheric VLF propagation on the basis of finite element method. Radio Science 30, 1511-1517.

1984 Effect of a heated patch of auroral ionosphere on VLF radio wave propagation. Nature 309, 6825-6832.

1985 The diffraction of VLF radio waves by a patch of ionosphere illuminated by a powerful HF transmitter. $J$. Geophysical Research 90, 2861-2875.

1961 Radio Waves in the Ionosphere. Cambridge University Press, New York.

1982 A study of whistlers correlated with bursts of electron precipitation near $L=2$. J. Geophysical Research 87, $4427-4434$.

1984 Use of subionospheric Siple transmitter signals to study burst precipitation outside the plasmapause. Antarctic J. of the US 19, 220-222.

1985 A new VI.F method for studying hurst precipitation near the plasmapause. J. Geophysical Research 90, 4383-4388.

1987 Seasonal, latitudinal and diurnal distributions of whistler-induced electron precipitation events. J. Geophysical Research 92, 3429-3435.

1990 Radio propagation and circuit performance at frequencies below about $30 \mathrm{kHz}$. Document 6/283,6/175. Report 895-1.

1990 Quasi-periodic particle precipitation and associated Trimpi activity observed at Halley Antarctica. $J$. Atmospheric and Terrestrial Phys. 52, 365-375.

Signature of burst particle precipitation on VLF signals propagating in the Antarctic Earth-Ionosphere waveguide. J. Geophysical Research 96, 19375-19387.

Phase and amplitude perturbations on subionospheric signals explained in terms of echoes from lightning induced electron precipitation ionization patches. $J$. Geophysical Research 93, 11543-11550.

1989a Modal effects on amplitude perturbations on subionospheric signals (Trimpis) deduced from two frequency measurements. J. Geophysical Research 94, $1515-1519$.

1989b Phase and amplitude perturbations on the NWC signal at Dunedin from lightning induced electron precipitation. I. Geophysical Research 94, 497-503.

1990 Location of lightning induced electron precipitation from measurements of VLF phase and amplitude perturbations on spaced antennas and on two frequencies. J. Geophysical Research 95, 4135-4145.

1991 Phase and amplitude perturbations on subionospheric signals produced by a moving patch of artificially heated ionosphere. J. Geophysical Research 96, 239 248. 
Dowden R. L. and Adams C. D. D.

Dowden R. L. and Adams C. D. D.

Dowden R. L., Adams C. D. D., Brundell C. B. and Dowden P. E.

Friedel R. H. W. and Hughes A. R. W.

Friedel R. H. W. and Hughes A. R. W.

Friedel R. H. W., Hughes A. R. W., Dowden R. L. and Adams C. D. D.

Helliwell R. A., Katsufrakis J. P. and Trimpi M. L.

Hurren P. J., Smith A. J., Carpenter D. L. and Inan U. S.

Inan U. S., Carpenter D. L., Helliwell R. A. and Katsufrakis J. P.

Inan U. S. and Carpenter D. L.

Inan U. S. and Carpenter D. L.

Inan U. S., Burgess W. C., Wolf T. G., Shafer D. C. and Orville, R. E.

Inan U. S., Shafer D. C., Yip W. Y. and Orville R. E.

Inan U. S., Wolf T. G. and Carpenter D. L.

Inan U. S., Knifsend F. A. and Oh J.

Inan U. S.

Inan U. S. and Rodriguez J. V.

Leyser T. B., Inan U. S., Carpenter D. L. and Trimpi M. L.

Lohrey B. and Kaiser A. B.

Morfitt D. G. and Shellman C. H.

Nickolaenko A. P.

1994

Pappert R. A., Gossard E. E. and Rothmuller I. J. 1993a Size and location of lightning induced enhancements from measurements of VLF phase and amplitude perturbations on multiple antennas. $J$. Atmospheric and Terrestrial Phys. 55, 1335-1359.

1993b VLF holography. The RadioScientist 4(3), 60-65.

1994 Rapid Onset, rapid decay (RORD) phase and amplitude perturbations on VLF subionospheric transmissions. J Atmospheric and Terrestrial Physics 56, 1513-1527.

1990 Characteristics and frequency of occurrence of Trimpi events recorded during 1982 at Sanae, Antarctica. $J$. Atmospheric and Terrestrial Phys. 52, 329-339.

1992 Trimpi events on low latitude paths: an investigation of gyroresonance interactions at low $\mathrm{I}$, values. $J$. Atmospheric and Terrestrial Physics 54, 1375-1386.

1993 First observations of Trimpi events at Durban using an OMSKI receiver. J. Geophysical Research 98, $1571-$ 1580 .

1973 Whistler induced perturbation in VLF propagation. $J$. Geophysical Res. 78, 4679-4688.

1986 Burst precipitation induced perturbations on multiple VLF propagation paths in Antarctica. Annales Geophysicae 4A, 311-318.

1985 Subionospheric VLF/LF phase perturbations produced by lightning whistler induced particle precipitation. J. Geophysical Research 90, 7457-7469.

1986 On the correlation of whistlers and associated subionospheric VLF/LF perturbations. J. Geophysical Res. 91, 3106-3116.

1987 Lightning induced electron precipitation events observed at $\mathrm{L}=2.4$ as phase and amplitude perturbations of subionospheric VLF signals. $J$. Geophysical Research 92, 3292-3303.

1988 a Lightning associated precipitation of $\mathrm{MeV}$ electrons from the inner radiation belt. Geophysical Research Letters 15, 172-175.

1988b Subionospheric VLF signatures of night time D region perturbations in the vicinity of lightning discharges. I. Geophysical Research 93, 11455-11477

$1988 \mathrm{c}$ Geographic distribution of lightning induced electron precipitation observed as VLF/LF perturbation events. J. Geophysical Res. 93, 9841-9853.

1990 Subionospheric VLF imaging of lightning induced electron precipitation from the magnetosphere. J. Geophysical Research 95, 17217-17231.

1990 VLF heating of the lower ionosphere. Geophysical Research Letters 17, 729-732.

1993 Lightning induced effects on VLF/LF Radio propagation, In AGARD Conference Proceedings CP-529: ELF/VLF/LF radio propagation and system aspects, NATO, Neuilly sur Seine, France, 9-1:9-10.

1984 Diurnal variation of burst precipitation effects on subionospheric VLF/LF signal propagation near $L=2 . J$. Geophysical Research 89, 9139-9143.

1979 Whistler induced anomalies in VLF propagation. $J$. Geophysical Research 84, 5122-5130.

1976 MODESRCH, an improved computer program for obtaining ELF/VLF/LF mode constants, Naval Electronics Lab. Centre Interim Report 771, NTIS Accession No ADA032573. National Technical Information Service, Va 22161, USA.

ELF radio wave propagation in a locally nonuniform Earth-ionosphere cavity. Radio Science 29, 11871199.

1967 A numerical investigation of classical approximations used in VLF propagation. Radio Science 2, 387--399. 
560

Pappert R. A.

Pappert R. A.

Pappert R. A. and Bickel J. E.

Pappert R. A. and Hitney L. R.

Poulsen W. L., Bell T. F. and Inan U. S.

Poulsen W. L., Bell T. F. and Inan U. S.

Poulsen W. L., Inan U. S. and Bell T. F.

Rycroft M. J.

Rycroft M. J.

Smith A. J. and Cotton P. D.

Smith A. J., Cotton P. D. and Robertson J. S.

Smith A. J.

Strangeways H. J.

Wait J. R. and Spies K. P.

Wait J. R.

Wait J. R.

Wait J. R.

Wait J. R.

Wait J. R.

Wait J. R.

Wolf T. G. and Inan U. S.
D. Nunn

1968 A numerical study of VLF mode structure and polarisation below an anisotropic ionosphere. Radio $\mathrm{Sci}$ ence 3, $219-233$.

1970 Effects of elevation and ground conductivity on horizontal dipole excitation of the earth-ionosphere waveguide. Radio Science 5, 579-590.

1970 Vertical and horizontal VLF fields excited by dipoles of arbitrary orientation and elevation. Radio Science 5, 1445-1452.

1988 Empirical modelling of nighttime easterly and westerly VLF propagation in the earth-ionosphere waveguide. Radio Science 23, 599-611.

1990 Three dimensional modelling of subionospheric VLF propagation in the presence of localised $\mathrm{D}$ region perturbations associated with lightning. $J$. Geophysical Research 95, 2355-2366.

1993a The scattering of VLF waves by localised ionospheric disturbances produced by lightning induced electron precipitation. J. Geophysical Research 98, 1555315559.

1993b A multiple mode three dimensional model of VLF propagation in the earth-ionosphere waveguide in the presence of localised D region disturbance. $J$. Geophysical Research 98, 1705-1717.

1991 Interactions between whistler mode waves and energetic electrons in the coupled system formed by the magnetosphere, ionosphere and atmosphere. $J$. Atmospheric and Terrestrial Physics 53, 849-858.

1993 A review of whistlers and energetic electron precipitations. In Review of Radio Science 1990-1992, ed W.R. Stone and G. Hyde, Oxford University Press, Oxford, 631-642.

1990 The Trimpi effect in Antarctica: Observations and models. J. Atmospheric and Terrestrial Phys. 52, 341-355.

1993 Transient $(\sim 10 s)$ VLF amplitude and phase perturbations due to lightning induced electron precipitation into the ionosphere (the 'Trimpi effect'), in AGARD Conference Proc., CP-259: ELF/VLF/LF Radio Propagation and Systems Aspects, NATO, Neuilly sur Seine, France, 8.1-8.7.

1996 Aspects of wave-particle interactions at mid-latitudes. Adv. Space Res. 17, 10213-10222.

1996 Lightning Trimpis and Sprites. Reviews of Radio Science, 1993-1996 (Stone R., ed.), Oxford University Press, Oxford, pp. 741-780.

1964 Characteristics of the earth-ionosphere waveguide for VLF radio waves, NBS Tech. Note, 300 .

1964 Influence of a circular ionospheric depression on VI.F propagation. Radio Science 68D, 907-914.

1964a On phase changes in VLF propagation induced by an ionospheric depression of finite extent. J. Geophysical Research 69, 441-446.

1970 Electromagnetic Waves in Stratified Media. Pergamon, New York.

1980 Electromagnetic scattering from an isolated irregularity in a tropospheric duct. Acta Physica Austriaca 52, $193-202$.

1991 VLF radio wave mode conversion for ionospheric depressions. Radio Science 26, 1261-1265.

1995 VLF scattering from a column of ionization in the earth-ionosphere waveguide. J. Atmospheric and Terrestrial Physics 57, 955-959.

1990 Path dependent properties of subionospheric VLF amplitude and phase perturbations associated with lightning. J. Geophysical Research 95, 20997-21005. 